\title{
Acetyltransferase TIP60/KAT5 regulates the Drosophila Circadian Clock
}

\author{
Dissertation \\ for the award of the degree \\ "Doctor rerum naturalium" \\ of the Georg-August-Universität Göttingen \\ within the doctoral program Genes and Development \\ of the Georg-August University School of Science (GAUSS)
}

submitted by

\section{Vinodh Ilangovan}

from

Tiruchirappalli, Tamil Nadu, India

Göttingen, 2015 


\section{Thesis Committee}

Prof. Dr. Gregor Eichele, Genes and Behavior, Max-Planck-Institute for Biophysical Chemistry, Göttingen (Mentor and Reviewer)

Prof. Dr. André Fiala, Molecular Neurobiology of Behavior, Schwann-Schleiden Research Centre, Göttingen (Co-reviewer)

PD. Dr. Halyna Shcherbata, Gene Expression and Signaling Group, Max-PlanckInstitute for Biophysical Chemistry, Göttingen

\section{Members of the Extended Examination Board}

Prof. Dr. Gregor Bucher, Developmental Biology, Blumenbach Institute, Georg-August-University, Göttingen

Dr. Manuela Schmidt, Emmy Noether-Research Group Somatosensory Signaling Max Planck Institute for Experimental Medicine, Göttingen

Prof. Dr. JörgGroßhans, Institute of Biochemistry and Molecular Cell Biology, University Medical Center, Göttingen

Date of oral examination: 13.10.2015 


\begin{abstract}
Affidavit
I hereby declare that the dissertation entitled "Acetyltransferase TIP60/KAT5 regulates the Drosophila Circadian Clock" is submitted in support of my application for the degree of Doctor rerum naturalium to Georg-August-Universität Göttingen within the doctoral program Genes and Development of the Georg-August University School of Science (GAUSS). This thesis has been written independently and with no other sources and aids other than quoted within texts, references and acknowledgments. This thesis has not been submitted in any previous application for any degree, elsewhere.
\end{abstract}

Göttingen, August 2015

Vinodh Ilangovan 


\section{எப்பொருள் யார்யார்வாய்க் கேட்பினும் அப்பொருள்}

மெய்ப்பொருள் காண்ப தறிவு. (குறள்: 423)

Whatever may be the source of information, knowledge is to discern and extract the truth in it.

(Tirukkural: 423) 


\section{Table of Contents}

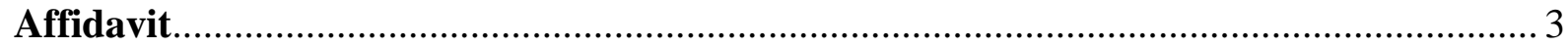

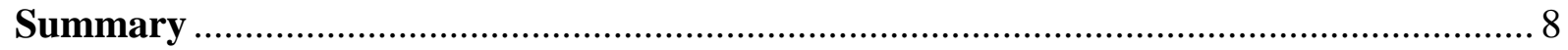

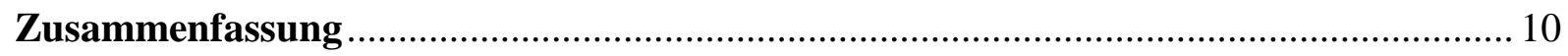

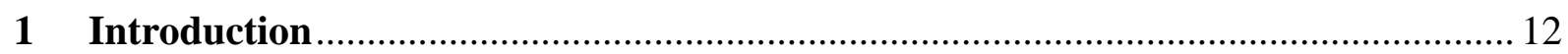

1.1 Circadian clock work at the molecular level ........................................................ 13

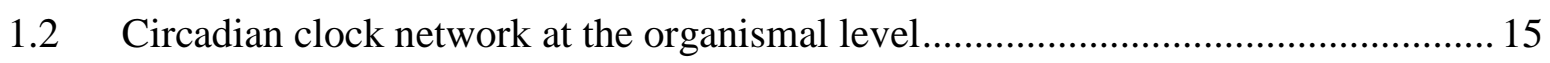

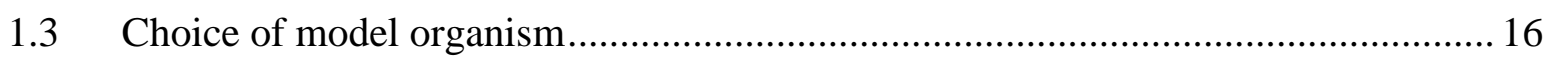

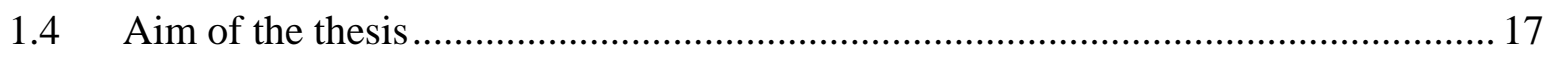

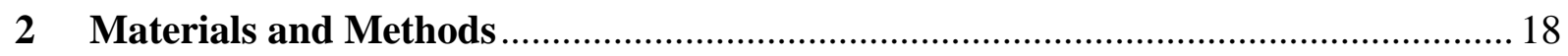

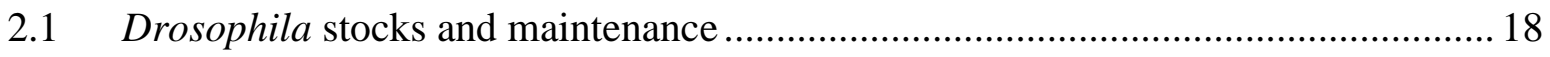

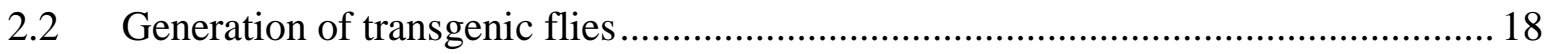

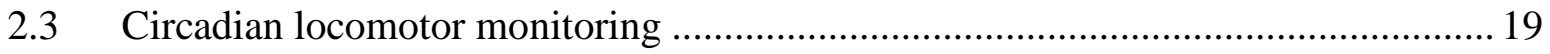

2.4 RNA extraction and Quantitative real time PCR ...................................................... 19

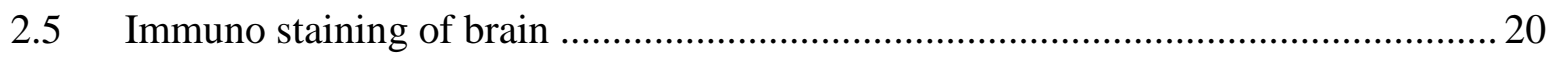

2.6 Chromatin immunoprecipitation (ChIP) ...................................................... 21

2.7 Co-immunoprecipitation using fly protein extracts .......................................... 22

2.8 Western blotting of fly protein extracts ........................................................ 22

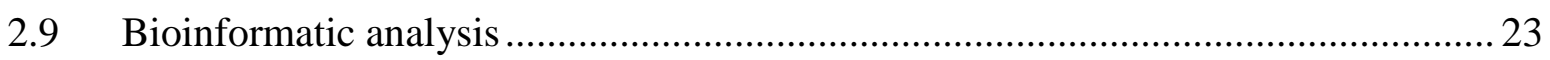

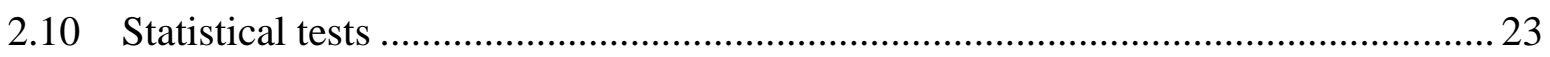

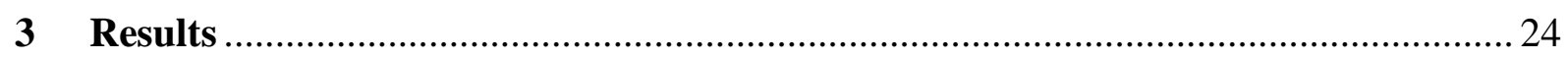

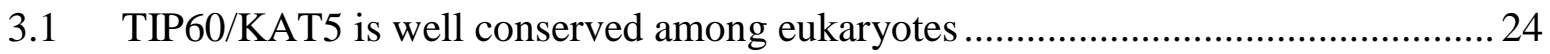

3.2 TIP60/KAT5 localizes in circadian pacemaker neurons ...................................... 27

3.3 Tools to manipulate TIP60/KAT5 levels in circadian pacemaker neurons .............. 28

3.4 Validation of spatio-temporal TIP60 knockdown ............................................. 28

3.5 Adult lifespan is mediated by TIP60 in lateroventral clock neurons ...................... 31 
3.6 TIP60/KAT5 is required in adult pacemaker clock neurons for robust circadian behavioral rhythmicity

3.7 Overexpression of $T I P 60^{E 431 Q}$ in adult clock neurons does not impair PDF neuropeptide expression and the morphology of pacemaker neuronal network 36

3.8 Expression of core clock controlled genes are modulated by TIP60_........................ 38

3.9 Tip60 mRNA does not oscillate.

3.10 Subunits of Nu4A multimeric protein complex that interact with TIP60 contribute to robustness in behavioral rhythm.

3.11 Tip60 genetically interacts with Cyc to regulate circadian rhythms..... 46

3.12 Generation and validation of transgenic strains to express dTIP60-HA tag as a fusion protein 49

3.13 TIP60 localizes near CLK/CYC binding sites on clock controlled genes 51

3.14 TIP60 interacts with CYC in vivo. 54

4 Discussion. 55

4.1 Ubiquitous presence of TIP60 and a role in circadian clock. 55

4.2 Establishing TIP60 in the circadian molecular loop 56

4.3 Lysine acetylation in CYC as a potent regulator of circadian transcription 59

4.4 Functions of TIP60 converging into the circadian clock 60

4.5 Integrating TIP60 within the network model of circadian clock 61

5 References 63

Acknowledgements 69 


\section{List of Figures}

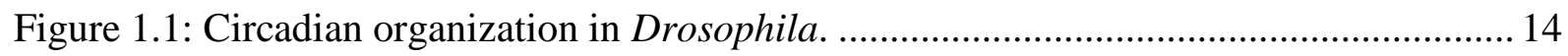

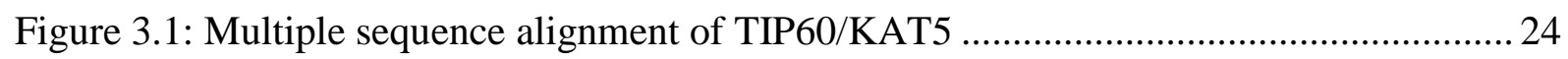

Figure 3.2: Transcriptome profile of Tip60 obtained from modENCODE data ..................... 26

Figure 3.3: TIP60/KAT5 is expressed in clock neurons of adult Drosophila brain ............... 27

Figure 3.4: Validation of spatio-temporal Tip60 knockdown using TARGET ........................ 30

Figure 3.5: Targeted knockdown and misexpression of dTIP60 in pacemaker neurons using

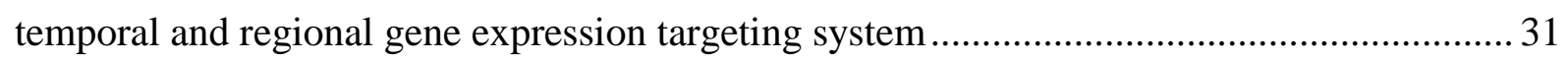

Figure 3.6: TIP60 in pacemaker clock neurons is required for healthy adult survival ............ 32

Figure 3.7:TIP60/KAT5 mutants have aberrant free running rhythms .................................. 35

Figure 3.8: Spatiotemporal expression of $T I P 60^{E 431 Q}$ in pacemaker clock neurons does not

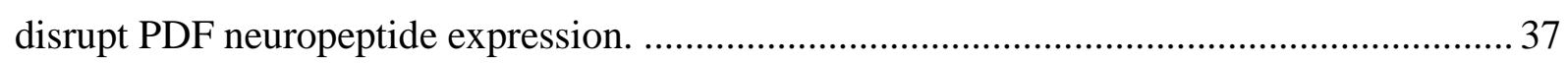

Figure 3.9: Transcriptional effects of TIP $60^{\mathrm{E} 431 \mathrm{Q}}$ misexpression in the circadian clock ......... 40

Figure 3.10: TIP6O ${ }^{E 431 Q}$ overexpression strongly dampens PER oscillations in clock neurons

Figure 3.11: Tip60 mRNA is not controlled by the circadian clock .43

Figure 3.12: Subunits of Nu4Achromatin-remodeling multi protein complex that includes TIP60, contributes to the robust regulation of circadian behavioral rhythms ......................... 45

Figure 3.13: Genetic interaction of TIP60 with core clock components................................ 48

Figure 3.14: Characterization of transgenic strain for the expression of tagged TIP60...........50

Figure 3.15: TIP60 localizes to CLK/CYC binding sites on Per and Tim genes with spatial

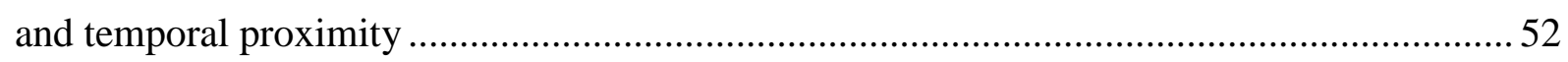

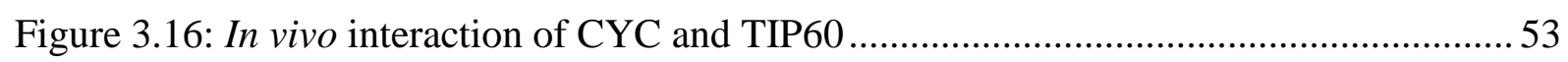

Figure 4.1: A model of TIP60 induced activation of circadian clock and balance by repressors 56

Figure 4.2: Pair-wise sequence alignment of mouse BMAL1 and Drosophila CYCLE ......... 58

Figure 4.3: Proposed manipulations of circadian network in Drosophila brain ..................... 61 


\section{Summary}

\section{Acetyltransferase TIP60/KAT5 regulates the Drosophila Circadian Clock}

Endogenous clocks control behavioral and physiological processes in a rhythmic fashion exhibiting periodicity of $\sim 24 \mathrm{~h}$. Drosophila exhibits a robust rhythm in activity/rest patterns which is controlled by circadian pacemaker neurons situated in the brain. Innate rhythms of gene expression are generated by the translational transcriptional positive and negative feedback driving the circadian clock. Although the feedback regulation of clock genes is relatively well understood, the factors that stimulate activators to start a new circadian cycle remain unclear. Post-translational modification of specific proteins by acetylation facilitates gene transcription. TIP60/KAT5 is a member of the well conserved MYST family of acetyltransferases and is involved in a variety of cellular processes such as cell growth and DNA repair by acetylating several target proteins. The main goal of this work is to investigate the mechanisms by which lysine acetyltransferase TIP60/KAT5 orchestrates the circadian clock of Drosophila.

UAS/GAL4 binary system was utilized to achieve targeted knock down (by RNA interference) or overexpression (a mutation in the catalytic residue rendering dominant negative function) of TIP60/KAT5 in circadian pacemaker neurons. The temperature sensitive GAL80 (TARGET) system was used to allow proper development of pacemaker neurons until adulthood. Non-invasive locomotor activity assays demonstrated that TIP60 in pacemaker neurons is required for robust circadian rhythm in the absence of time cues during adulthood. Consistent with the observed changes in circadian locomotor behavior, rhythmic expression of key clock controlled genes such as Period and Timeless as well as auxiliary loop genes Vrille and Clockwork orange was dampened. However, Tip60 mRNA was found to be regulated independently of the clock. Circadian locomotor behavior was employed to assess if individual subunits of Nu4A multimeric complex also participate in regulation of rhythm. In order to determine the targets of TIP60 in the circadian clock, a genetic interaction assay using adult circadian locomotor activity phenotype was performed and a strong interaction with the circadian co-activator CYCLE and the repressor PERIOD was found in transheterozygous crosses. 
Due to non-availability of a specific antibody to study the function of TIP60 in vivo in adult Drosophila, a transgenic strain (UAS dTIP60-HA) with hemagglutinin epitope tag was generated. This transgenic strain was used to overexpress TIP60-HA using a ubiquitous promoter (tub GAL4). Using an antibody against the epitope (anti-HA) chromatin immuno-precipitation (ChIP) was performed in these transgenic flies. TIP60 was found to be localized rhythmically in the regulatory region of clock controlled genes, indicating a rhythmic occupancy at DNA elements similar to CLOCK/CYCLE complex. Co-immunoprecipitation was performed with transgenic flies to demonstrate protein-protein interaction of TIP60 and CYCLE. These results further validate the genetic interaction between TIP60 and CYCLE. The findings of this study clearly indicate a role of TIP60/KAT5 as a co-activator in the precise regulation of Drosophila circadian clock. 


\section{Zusammenfassung}

\section{Die Acetyltransferase TIP60/KAT5 steuert die circadiane Uhr in Drosophila}

Endogene Uhren steuern verhaltensbezogene und physiologische Prozesse in einer rhythmischen Weise, die eine Periodizität von $\sim 24 \mathrm{~h}$ aufweist. Drosophila zeigt einen robusten Rhythmus von Aktivitäts- /Ruhephasen, der von im Gehirn befindlichen zirkadianen Schrittmacherneuronen gesteuert wird. Angeborene Rhythmen der Genexpression werden durch positive und negative transkriptionelle/translationale Rückkopplungsschleifen, die die circadiane Uhr antreiben, generiert. Obwohl die Regulierung der Rückkopplung von Uhrengenen relativ gut verstanden ist, sind die Faktoren, die die Aktivatoren stimulieren, einen neuen circadianen Zyklus zu beginnen, noch unbekannt. Posttranslationale Modifikationen spezifischer Proteine durch Acetylierung erleichtert die Gentranskription. TIP60/KAT5 ist ein Mitglied der hochkonservierten MYSTFamilie von Acetyltransferasen und ist an einer Vielzahl zellulärer Prozesse beteiligt, wie beispielsweise Zellwachstum und DNA-Reparatur, undzwar indem es verschiedene Zielproteine acetyliert. Das Hauptziel dieser Arbeit ist es, die Mechanismen, durch die LysinAcetyltransferase TIP60/KAT5 die circadiane Uhr von Drosophila steuert, zu untersuchen.

Das binäre UAS/GAL4 System wurde eingesetzt, um einen gezielten knock down (durch RNAInterferenz) oder eine Überexpression (eine Mutation in der für die Katalyse essentiellen Aminosäure, wodurch eine dominant negative Funktion verursacht wird) von TIP60/KAT5 in zirkadianen Schrittmacherneuronen zu erreichen. Das temperatursensitive GAL80 (TARGET) System wurde verwendet, um die normale Entwicklung der Schrittmacherneuronen bis ins Erwachsenenstadium zu ermöglichen. Unter Verwendung von nicht-invasiven lokomotorischen Aktivitätstests konnte gezeigt werden, dass TIP60 in Schrittmacherneuronen für den robusten zirkadianen Rhythmus in Abwesenheit von Zeitgebern im adulten Stadium erforderlich ist. In Übereinstimmung mit den beobachteten Veränderungen des circadianen Bewegungsverhaltens, war die rhythmische Expression von wichtigen clock-controlled genes wie Period und Timeless sowie Genen der Hilfsschleife Vrille und Clockwork orange gedämpft. Im Gegensatz dazu konnte gezeigt werden, dass die Tip60 mRNA unabhängig von der Uhr reguliert wird. Das zirkadiane Bewegungsverhalten wurde genutzt, um zu beurteilen, ob Untereinheiten des TIP60 
(Nu4A) multimeren Komplexes auch an der Regulation des Rhythmus beteiligt sind. Um die Zielproteine von TIP60 in der circadianen Uhr zu bestimmen, wurde ein genetischer Interaktions-Test unter Verwendung des Phänotyps der circadianen Lokomotoraktivität durchgeführt und es wurde eine starke Interaktion mit dem zirkadianen Co-Aktivator CYCLE und dem Repressor PERIOD in trans-heterozygoten Kreuzungen gefunden.

Aufgrund des Fehlens eines spezifischen Antikörpers, um die Funktion von TIP60 in vivo in adulter Drosophila zu untersuchen, wurde ein transgener Stamm (UAS dTIP60-HA) mit einem Hämagglutinin-Epitop-Tag erzeugt. Dieser transgene Stamm wurde genutzt, um TIP60-HA unter Verwendung eines ubiquitären Promotors (tub GALA) überzuexprimieren. Ein Antikörper gegen das Epitop (Anti-HA) wurde verwendet, um einen Chromatin-Immunopräzipitations-Assay (ChIP) in diesen transgenen Fliegen durchzuführen. Es zeigte sich, dass TIP60 rhythmisch in der regulatorischen Region von clock-controlledgenes lokalisiert ist, was eine rhythmische Belegung von DNA-Elementen, ähnlich dem CLOCK/CYCLE Komplex, nahelegt. Co-Immunpräzipitation wurde mit transgenen Fliegen durchgeführt, um eine Protein-Protein-Wechselwirkung zwischen TIP60 und CYCLE nachzuweisen. Die Ergebnisse dieser Studie legen deutlich eine Beteiligung von TIP60/KAT5 als Co-Aktivator an der präzisen Steuerung der circadianen Uhr in Drosophila nahe.

Deutsche Übersetzung: Dr. Inga Urban 


\section{Introduction}

All organisms adapt efficiently to changes occurring in their environment over the time of day for maximizing their survival. To deal with such challenges, organisms have evolved fundamental mechanisms such as circadian clocks that prepare them to cope with daily environmental changes (Aschoff, 1963). Circadian (Circa = approximate; dies = a day) oscillations are well documented at different levels of biological organization starting from subcellular molecules, intercellular communication, tissues to individual organisms and at population level in behavioral, physiological, and metabolic processes in a variety of organisms (Zordan et al., 2000). The circadian clock is an evolutionarily conserved genetic circuit that controls innate oscillations of behavior and physiology in a fashion exhibiting periodicity of $\sim 24$ h. Circadian clocks have an ubiquitous occurrence, being present on most organisms from cyanobacteria to human beings (Johnson, 2004; Bell-Pedersen et al., 2005). This suggests that they may have an adaptive significance, but, this adaptive advantage of circadian organization is not yet substantiated by many experimental evidences. The functional roles of the biological clocks have been studied to quite an extent in various organisms. It is generally agreed that it is advantageous to have temporal orchestration and, where needed, segregation of various physiological and behavioral processes (DeCoursey, 2004). The clocks also help in anticipation of daily as well as seasonal changes in the environment. It may also have importance in general well-being or, as a consequence, even overall longevity of the organism (Kumar et al., 2005).

Although circadian clocks are present in both prokaryotes and eukaryotes at functionally analogous manner, they differ in their homology of constituents suggesting convergent evolution (Bell-Pedersen et al., 2005). However, the high degree of conservation found in the genes constituting molecular clocks within the animal kingdom suggests that these clocks must have possibly evolved from a common progenitor. This can be demonstrated with the example of similarity between circadian clock genes in an early evolved invertebrate Drosophila and relatively recently evolved mammal mouse. Gene duplication events in the vertebrate branch could be attributed to the fact that in mammals most clock genes exist in at least two copies, whereas in Drosophila there is a single homolog for each respective clock gene (Looby and Loudon, 2005). 


\subsection{Circadian clock work at the molecular level}

The molecular mechanism of the circadian clock works cell-autonomously and is based on interlocking auto-regulatory transcriptional-translational feedback loops (TTFLs). A mutagenesis screen identified different alleles of the Period gene leading to either arrhythmic, short period or long period eclosion rhythms in fruit fly Drosophila (Konopka and Benzer, 1971). This was the first identified genetic basis for circadian clock. Studies in Drosophila melanogaster have been instrumental in understanding the mechanisms of clock in general. In Drosophila, the transcription factors dCLOCK (dCLK) and CYCLE (CYC) act as a heterodimer (dCLK/CYC), promoting the transcription of the $\mathrm{d}$ Period (dPer) and timeless (Tim) genes. In mammals, the orthologs mCLK (or mNPAS2 in the forebrain) and mBMAL1 exert the function of positive regulators activating the transcription of the three mammalian orthologs of $\mathrm{d}$ Period (mPerl, mPer 2 , and mPer 3 ) and the two mCryptochrome genes (mCryl and mCry2). In mammals, the mCry genes replace Tim in the main TTFL (Zordan and Sandrelli, 2015). Once translated, dPER and TIM (mPERs and mCRYs in mammals) are targeted by different kinases and phosphatases, which mediate the timing of their nuclear translocation, stability, and action as negative feedback elements of $\mathrm{dCLK} / \mathrm{dCYC}$ (or mCLK/mBMAL1 in mammals) regulatory activity. Among the kinases, it is worth indicating the roles played by dSHAGGY, homologous to mammalian glycogen synthase kinase-3 (mGSK3), which is involved in the phosphorylation of dTIM and dPER (mPERs and mCRYs in mammals), and by dDOUBLETIME (dDBT), homologous to mammalian Casein Kinase $1 \varepsilon(\mathrm{CK} 1 \varepsilon)$, which targets dPER (Price et al., 1998). These factors are involved in the regulation of dPER and dTIM (mPERs and mCRYs in mammals) stability and nuclear entry and contribute to the fine-tuning of circadian rhythmicity. dCLK/CYC (mCLK/mBMAL1) are also the positive regulators of an auxiliary TTFL, which controls the rhythmic expression of $\mathrm{d} C l k$ in flies and mBmall in mammals. In Drosophila, this TTFL is under negative control by dVRILLE (dVRI), which competes with the positive regulator dPDP1 to bind sequence elements in the promoter region of dClk (Cyran et al., 2003). In mammals, the second TTFL is controlled by the nuclear hormone receptors mRORs and mREV-ERBs, which act as transcriptional repressors and activators of mBmall, respectively (Preitner et al., 2002; Sato et al., 2004). 

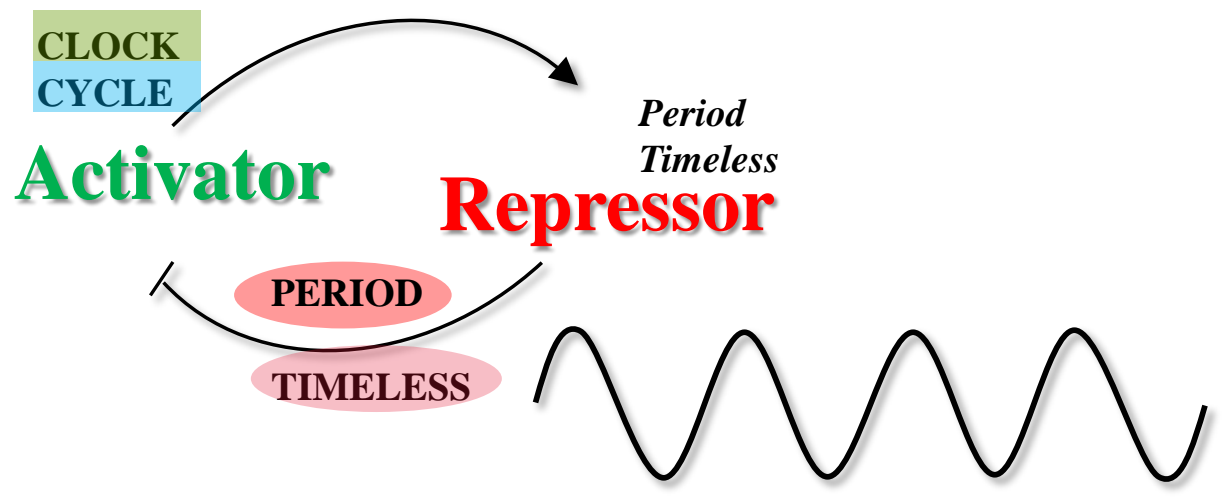

B

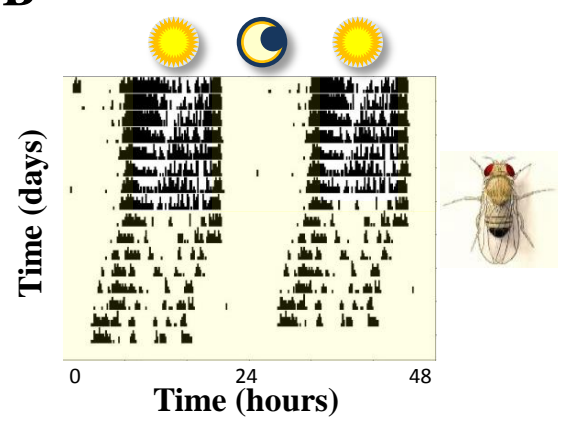

C

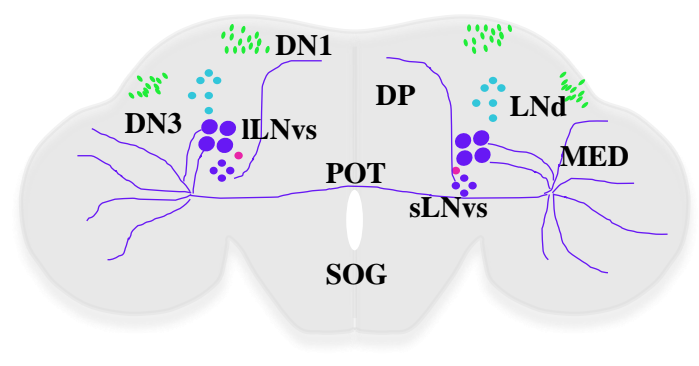

\section{Figure 1.1: Circadian organization in Drosophila.}

(A) Schema of transcriptional translational feedback loop (TTFL) generating periodic cycles. The components of Drosophila molecular clock are shown in their respective roles. (B) Recording locomotor activity is a robust and non-invasive method to infer the state of the circadian clock in a living animal and is often best portrayed in a vertical stack of $48 \mathrm{~h}$ traces called double plotted actograms. Activity measurements are done using monitoring tubes for flies. Vertical bars represent activity; yellow shading denotes experimental dark phases. Flies are diurnal species and when released in constant darkness they start to show their intrinsic period, also known as "free-running period" of circadian rhythms in the absence of external cues. (C) Anatomical organization of circadian pacemaker network in Drosophila brain. Anatomical locations of POT- Protocerebral tract, MED- Medullary projection, DP-dorsal projection, ILNvs- large Lateroventral neurons, sLNvs- small Lateroventral neurons, DN- dorsal neurons, LNd- Laterodorsal neurons, SOG- Sub esophageal Ganglion. 


\subsection{Circadian clock network at the organismal level}

Adult Drosophila exhibit robust rhythm in activity/rest patterns which is controlled by circadian pacemaker neurons situated in the brain. In contrast to the mammalian master pacemaker located in hypothalamic suprachiasmatic nuclei $(\mathrm{SCN})$, central pacemaker in Drosophila is distributed across multiple groups of clock neurons in the brain (Yao and Shafer, 2014). Apart from initiating the molecular feedback loop, CLK can also induce ectopic clocks in non-circadian tissues upon overexpression (Zhao et al., 2003). CLK expression is detected in a subset of clearly identified pacemaker neurons during early development (Houl et al., 2008) which also exhibits oscillation of PER (Kaneko et al., 1997) and hence are classified as early pacemaker neurons (small ventral lateral neurons-sLN $\mathrm{v}_{\mathrm{v}} \mathrm{s}$, anterior dorsal neurons group 1- $\mathrm{DN}_{1 \mathrm{a}} \mathrm{S}$ and dorsal neurons group 2-DN $2 \mathrm{~S}$ ). Rest of the population constitute the major quantum of pacemakers identified in adulthood that display rhythm in PER expression preluding from late metamorphosis and therefore known as late pacemaker neurons (dorsal lateral neurons- $\mathrm{LN}_{\mathrm{d}} \mathrm{S}$, posterior dorsal neurons-DN $\mathrm{DN}_{1 \mathrm{p}} \mathrm{s}$, lateral posterior neurons-LPNs, dorsal neurons group 3-DN $\mathrm{DN}_{3} \mathrm{~s}$, large ventral lateral neurons-lLN $\mathrm{L}_{\mathrm{v}}$,) (Kaneko et al., 1997). These pacemaker neurons are present in all consecutive developmental stages (Kaneko et al., 1997; Houl et al., 2008) indicating the existence of circadian clocks during early development that might confer adaptive advantage to the organism. Emergence of adult imago from pupal case, which occurs once in the lifetime of an individual fly is under the control of circadian clock (Skopik and Pittendrigh, 1967; Myers et al., 2003).

In addition to the clock neurons, glial cells (in particular astrocytes) contribute to the daily control of locomotor activity rhythms (Suh and Jackson, 2007; $\mathrm{Ng}$ et al., 2011). In this activity, ebony, which encodes for an enzyme involved in dopamine and histamine recycling, seems to play a key role as an output gene (Suh and Jackson, 2007). Moreover, glia might act by modulating PDF transport and/or release from $\mathrm{LNv}$ projections, indicating the importance of a glia-to-neuron communication in the control of behavioral rhythmicity.

As in mammals, non-central-brain peripheral circadian clocks are present in multiple regions of the Drosophila body, such as the compound eyes, antennae (Krishnan et al., 1999), and prothoracic gland (Meyers et al. 2003). In the simplified model of the mammalian circadian 
system, the SCN master clock controls the phase of all the peripheral clocks (Husse et al., 2014), with the exception of the semi-autonomous oscillator in the olfactory bulb (Granados-Fuentes et al., 2004). This organization is not completely transposable to Drosophila. Experimental evidence instead suggests that in Drosophila, some peripheral clocks, such as those of the antenna (which control circadian odor-sensitivity), those of the proboscis (controlling the gustatory physiology rhythms), and those of the Malpighian tubules (the renal organ of the fly), are autonomous systems which might oscillate in phase with the master clock by directly perceiving and responding to the same environmental stimuli (Ivanchenko et al., 2001).

\subsection{Choice of model organism}

For the feasibility of carrying out the investigation of circadian clock, it is important to analyze the dysfunction using easily observable and quantifiable parameters. Drosophila is easy to maintain and has a short generation time exhibiting strong rhythms in activity/rest patters, eclosion from puparium, egg laying and olfactory learning. The Drosophila circadian clock is one of the best characterized at the molecular, physiological, and behavioral levels (Zordan and Sandrelli, 2015). Notwithstanding significant differences in the organization of the multipleclock system at the organismic level, the overall molecular architecture of the Drosophila and mammalian circadian oscillators are comparable and many components are evolutionarily conserved. GAL4 transcriptional activator was successfully adopted from yeast and used widely as the binary expression system in Drosophila to ectopically express transgenes of interest (Brand and Perrimon, 1993). The efficiency of this system has been further improved by optimizing the expression restricted in a spatio-temporal manner using a temperature sensitive GAL80 which inhibits the activity of GAL4. Therefore, by combining the UAS/GAL4 system and the Gal80ts (TARGET system), the gene of interest can be expressed in specifically in cells at a particular time window (McGuire et al., 2004). 


\subsection{Aim of the thesis}

Although the core transcriptional translational feedback loop (TTFL) of time keeping machinery appears to be conserved in eukaryotes, the tasks associated with operation of endogenous clocks is challenging to compute into simple models. For example, abundance or persistence of certain clock components during the circadian cycle greatly varies across animals such as insects and mammals. One such example is the constitutive expression of CYCLE (CYC), the fly homolog of rhythmically expressed mammalian transactivator BMAL1. This raises ambiguity on precise temporal regulation of the circadian clock thereby requiring additional rhythmic mediator. Extensive studies had focused on the regulation of the feedback loop, however how activation is induced remains very unclear. TIP60/KAT5 is a member of the well conserved MYST family of acetyltransferases and is involved in a repertoire of cellular processes such as cell growth and DNA repair by acetylating several target proteins. TIP60 (Tatinteracting protein of $60 \mathrm{kDa}$ ) was initially identified by a yeast two hybrid screen as a binding partner of the HIV1 Tat protein (Kamine et al., 1996; Creaven et al., 1999). TIP60 is the only known catalytic subunit of the evolutionarily conserved NuA4 (Nucleosome Acetyltransferase of histone H4) complex, which acetylates histones $\mathrm{H} 4$ and $\mathrm{H} 2 \mathrm{~A}$ in chromatin (Ikura et al., 2000) and hence turns on gene transcription.TIP60 is also known as lysine acetyltransferase 5 (KAT5) in accordance with nomenclature of enzymes, as it acetylates several non-histone substrates such as ATM and p53.

In order to tease out the functional link between activation of circadian gene transcription and TIP60, Drosophila circadian clock was chosen for investigation. Drosophila is an attractive model to study the regulation of circadian clock by TIP60 because the key molecular players of clock machinery are conserved and at the same time possess subtle differences in structure and function. The strong conservation of TIP60/KAT5 among eukaryotes also makes this investigation worthy to probe into the basic molecular underpinning of the circadian clock with respect to This study is an attempt to understand the functional link of lysine acetyltransferase dTIP60/KAT5 in the core clock machinery. 


\section{Materials and Methods}

\subsection{Drosophila stocks and maintenance}

Flies were maintained on standard corn meal under 12:12 h light and dark (LD) cycles at $25^{\circ} \mathrm{C}$ and $60 \%$ relative humidity, unless otherwise stated. Controlled temperature regimes and constant dark conditions during experiments were maintained with the help of an incubator (DigiTherm, Tritech Research Inc.) and improvised cubicles of 65 x65 x $65 \mathrm{~cm}$ constructed by the workshop personnel of Max Planck Institute of Biophysical Chemistry, Goettingen. The genotypes and rearing temperature conditions of experimental flies are appropriately described in the relevant results section. Handling of flies in dark conditions was assisted by a red lamp.

Per ${ }^{01}$ mutants described by (Konopka and Benzer, 1971) was obtained from Prof. Charlotte Helfrich-Foerster. $C y c^{01}$ mutants were a kind gift from Prof. Michael Rosbash. UAS$T I P 60^{E 431 Q}$ responder strain was kindly provided by Dr. Felice Elefant. One of the UAS-dTip60 RNAi (\#1) was provided by Prof. Ann. E. Ehrenhoeffer-Murray. All other fly stocks described were obtained from Bloomington Drosophila stock centre

\subsection{Generation of transgenic flies}

dTip60 coding region was amplified from a Drosophila cDNA pool using a forward primer

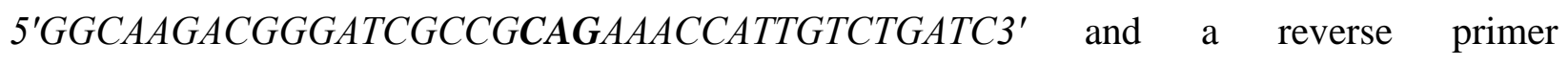
5'GATCAGACAATGGTTTCTGCGGCGATCCCGTCTTGCC3' respectively. The insert was subcloned into pRm3HA vector obtained from BIOSS Centre for Biological Signaling Studies, University of Freiburg. PCR reactions for strand synthesis step contained $25 \mathrm{ng}$ of dTip60 template DNA, $125 \mathrm{ng}$ each of forward and reverse primer, and Q5 High-Fidelty DNA polymerase (New England Biolabs) with master mix. The cycling parameters were 15 cycles of $95^{\circ}$ for 30 seconds, $55^{\circ}$ for 1 minute, and $68^{\circ}$ for 12 minutes using Mastercycler (Eppendorf). dTIP60 HA construct was subcloned into the pUASattB inducible expression vector obtained from University of Zurich. The full open reading frame (ORF) of $d T I P 60$ was amplified by PCR using forward primer 5'-CGG CGA ATT CGC CAA CAT GAA AAT TAA CCA CAA ATA TGA G- 
$3{ }^{\prime}$ containing an EcoRI site and a reverse primer 5'-GGT TGG TAC CTC ATC ATC ATT TGG $A G C$ GCT TGG ACC AGT $C$ - 3 ' containing a $\mathrm{KpnI}$ restriction site. PCR reactions were carried out with Q5 High-Fidelty DNA polymerase (New England Biolabs) using $400 \mathrm{nM}$ of each forward and reverse primer and cycling parameters: 30 cycles of $95^{\circ} \mathrm{C}$ for $2 \mathrm{~min}, 55^{\circ} \mathrm{C}$ for $1 \mathrm{~min}$, and $72^{\circ} \mathrm{C}$ for $4 \mathrm{~min}$, using a Mastercycler (Eppendorf). After ligation into the $p U A S a t t B$ vector, clones were selected and sequenced by Eurofins MWG for verification of final construct.

P-element germline transformations were performed by Best Gene Inc.(Chino hills CA, USA) from a total concentration $50 \mu \mathrm{g}$ (in a concentration of $1 \mu \mathrm{g} / \mu \mathrm{l}$ ) of $p$ UASattB-dTip60-HA construct. $200 w^{1118}$ fly embryos were injected and stable transformants were generated using Plan C Service "P-element Premium" to generate independent fly lines with balancers containing UAS-dTIP60-HA transgene.

\subsection{Circadian locomotor monitoring}

Individual flies of age 3 to 4 days old were housed in glass tubes with ad libitum access to food. Locomotor activity was measured using Drosophila Activity Monitors (Trikinetics) for 5 days in LD and 8 days in DD. Light and temperature controlled cubicles of 65 x65 x $65 \mathrm{~cm}$ constructed by the workshop in house were used for locomotor recordings. The periods were calculated by $\chi^{2}$ periodogram programmed using CLOCKLAB (Math Works Inc). Periods of rhythmic flies in each genotype is represented as mean \pm SEM. Rhythmic flies were defined by chi square periodogram analysis with the criteria of Power of rhythmicity not less than 20 at a significance level of 0.05 . Power and width are the height and width of the peak of the periodogram respectively.

\subsection{RNA extraction and Quantitative real time PCR}

Adult flies were collected at indicated time points and snap frozen in liquid nitrogen before exposure to light. These samples were labeled and stored at $-80{ }^{\circ} \mathrm{C}$ until use. The heads were mechanically separated by vortexing the snap frozen flies and passing them through a cooled sieve of $710 \mu \mathrm{m}$ to retain the thorax and body and with a $425 \mu \mathrm{m}$ sieve to retain the separated 
heads. Total RNA was extracted from 30 fly heads pooled from flies collected per genotype in four replicates using TRIZOL. cDNA was synthesized with Thermoscript (Invitrogen).

Quantitative real time PCR was performed on CFx96 Real time system (Bio-Rad) with samples containing master mix with IQ SYBR Green (Bio-Rad), $500 \mathrm{nM}$ forward and reverse primers and $1 \mathrm{ng}$ cDNA in $20 \mu \mathrm{l}$ reaction by the amplification procedure as follows: 40 cycles of $95^{\circ} \mathrm{C}$ for $15 \mathrm{sec}, 60^{\circ} \mathrm{c}$ for $30 \mathrm{sec}$ and $72^{\circ} \mathrm{C}$ for $45 \mathrm{sec}$. The set of forward and reverse primers used to amplify non conserved region of dTip60 are 5'-GACGGCTCACAAACAGGC-3' and 5'GGTGTTGCGGTGATGTAGG-3' respectively. Rpl32 which encodes for ribosomal protein L32 was used as internal control and the primer sets used to amplify are as follows: Forward 5'CTGCTCATGCAGAACCGCGT-3' and Reverse5'-GGACCGACAGCTGCTTGGCG-3'. Other primers for clock genes are described previously (McDonald and Rosbash, 2001; Kadener et al., 2008; Abruzzi et al., 2011; Meireles-Filho et al., 2014). Data was analyzed using Bio-Rad CFX Manager 3.0 software and the normalized values were plotted.

\subsection{Immuno staining of brain}

Adult flies were fixed with 4\% Paraformaldehyde and dissected in 1xPBS as described by (Hermann et al., 2013). Adequate washing was done with PBT (0.2\% Triton-100 in PBS) and blocked in 5\% normal goat serum. Primary antibodies used were mouse anti-PDF (1:50) (DSHB Hybridoma Bank), guinea pig anti-dTip60 (1:400) (gifted by Prof. Ehrenhofer-Murray), rat antiPER (1:2000) (gift from Prof. Amita Sehgal) and rabbit anti-HA (Pierce). After overnight incubation with primary antibody and adequate washes with PBT brains were incubated with secondary antibodies anti-mouse 488, anti-guinea pig 568, anti-rat 568 and anti-rabbit 556 (Abcam). After a brief wash with 1\% DAPI brains were mounted in slides with glycerol and NPG. Confocal images were also taken and analyzed as described by (Hermann et al., 2013). 


\subsection{Chromatin immunoprecipitation (ChIP)}

Chromatin immunoprecipitation was performed based on published protocols (MeirelesFilho et al., 2014) with modifications described. Flies entrained in 12:12 h, LD conditions at $25^{\circ} \mathrm{C}$ for five days were collected at four time-points (CT) on the fifth day. $500 \mu$ l volume of fly heads were homogenized with equal volume of ice cold $1 \%$ Formaldehyde in NE buffer $(15 \mathrm{mM}$ HEPES pH 7.6, 10mM KCl, 0.1mM EDTA, 0.5mM EGTA, 350mM Sucrose, 0.1\% Tween, $5 \mathrm{mM} \mathrm{MgCl}_{2}$, complete protease inhibitor cocktail [Roche]). Samples were sonicated 3x using a Diagenode Bioruptor on high setting for 5 minutes at 30 seconds on/off and then centrifuged at 10,000 rpm for 10 minutes. Subsequent steps such as quenching of cross linking and filtration was followed according to the protocol. The collected chromatin was immuno precipitated with anti-HA sepharose beads (Roche) overnight. Input samples were collected and stored for further analysis. Beads were then removed from IP samples and supernatant was de-crosslinked overnight at $65^{\circ} \mathrm{C}$. DNA was eluted using the Qiagen PCR purification kit and subjected to qPCR. The following primers were used to assay the presence of genomic regions from ChIP samples.

$\begin{array}{ll}\text { Per E-box forward } & \text { TGCCAGTGCCAGTGCGAGTTCG } \\ \text { Per E-box reverse } & \text { TGCCTGGTGGGCGGCTGG } \\ \text { Per 3' region forward } & \text { ACGACGAGATGGAGAAGTTCATGCTG } \\ \text { Per 3' region reverse } & \text { ATCCCGTGGCCTGGACCGCTG } \\ \text { Tim E-box forward } & \text { ACGTTGTGATTACACGTGAGCCG } \\ \text { Tim E-box reverse } & \text { TACACACACTGACCGAAACACCC } \\ \text { Tim 3' region forward } & \text { CCCTTATACCGAGGTGGAT } \\ \text { Tim 3' region reverse } & \text { TGATCGAGTTGCAGTGCTTC }\end{array}$

Background was subtracted and presented as the percentage of the input samples. For each assay, at least four biological replicates were performed, with technical duplicates for the qPCR step for each biological replicate. The technical qPCR duplicates were averaged for each biological replicate as no significant differences were found between them, and the error bars represent SEM calculated from variance between biological replicates. 


\subsection{Co-immunoprecipitation using fly protein extracts}

Transgenic flies expressing TIP60 fused to HA epitope tags were entrained in $12 \mathrm{hr}$ light:12 hr dark (LD) conditions at $25^{\circ} \mathrm{C}$ four days and collected on the next day of DD at CT 12 . Heads were separated using frozen metal sieves and homogenized in 3x volume of ice cold extraction buffer (20 mM HEPES at pH 7.5, $50 \mathrm{mM} \mathrm{KCl,} \mathrm{10 \%} \mathrm{glycerol,} 2 \mathrm{mM}$ EDTA, $1 \mathrm{mM}$ DTT, $1 \%$ Triton X-100, 0.4\% NP-40, complete protease inhibitor cocktail [Roche]). Homogenate was sonicated using a hand held sonicator for five seconds and repeated five times with 10-second pauses in between. Samples were spun down at 14,000 rpm for 15 minutes at $4^{\circ} \mathrm{C}$ to remove cell debris. Supernatant was collected, transferred to new tubes, and spun down again for 10 minutes at $14,000 \mathrm{rpm}$ at $4^{\circ} \mathrm{C}$. Supernatant was collected and protein levels were quantified using a Nano-Drop spectrophotometer. Extracts were quantified and equal concentrations were subjected for IP. Samples were pre-cleared using sepharose beads (Sigma) to reduce nonspecific binding.

Co-IPs were performed using $\alpha$-HA (Sigma) and $\alpha$-FLAG (Santa Cruz Biotechnology) antibodies. Samples were incubated with antibodies for overnight at at $4{ }^{\circ} \mathrm{C}$ on an end-over-end rotator. $20 \mu \mathrm{l}$ of GammaBind Plus sepharose beads (GE) was added and incubation was continued for 2 hours. Samples were washed with wash buffer (composition similar to extraction buffer but without protease inhibitors) three times, 10 minutes each, and immune complexes were resolved by SDS-PAGE (Criterion $7.5 \%$ gels, Bio-Rad) at $150 \mathrm{~V}$ for 40 minutes.

\subsection{Western blotting of fly protein extracts}

The Co-IP samples resolved on SDS-PAGE were transferred to prepacked nitrocellulose membranes (Bio-Rad) using Turbo Blot system (Biorad) using the default transfer options for TGX gels. Blocking was performed with $5 \%$ BSA in 1x TBST for one hour and antibody incubation was over-night at $4{ }^{\circ} \mathrm{C}$ with gentle shaking in a nutator. The following primary antibodies were used $\alpha$-HA (1:2000), $\alpha$-FLAG (1:2000), $\alpha$ - TUBULIN (Santa Cruz) (1:1000). Washing steps were 4 x 10 min in 1x TBST. The membrane was incubated with HRP-conjugated secondary antibodies (New England Biolabs) for two hours at room temperature. Protein band 
detection was performed using ECL substrates (Pierce) and the ImageQuant system (GE Healthcare).

\subsection{Bioinformatic analysis}

Protein sequences of TIP60/KAT5 were obtained from Uniprot (http://www.uniprot.org/). Multiple and pair-wise sequence alignment was performed using openly available tool BioEdit. RNA-seq reads for Tip60 from modEncode data (Contrino et al., 2012) was visualized with RNA-seq by Region tool from FlyBase (released on June 29, 2015) to get an overview of transcript expression during different developmental stages and in the adult heads under different treatment conditions.

http://flybase.org/static_pages/feature/previous/articles/2015_06/RNAseqByRegion.html

\subsection{Statistical tests}

GraphPad Prism Version 5.04 was used for all statistical analyses of the data. Error bars represent the standard error of the mean (SEM) in most cases of the data presented and are described in relevant sections. Various statistical tests used are described in relevant result sections. Two-way ANOVA was performed between parental genotypes and the mutants. In some cases of testing Tip60 knockdown efficiency, the error bars represent $95 \%$ confidence intervals of mean. Such variation in error bar representation was done to critically extract required information through visual inspection of the plots. 


\section{Results}

\subsection{TIP60/KAT5 is well conserved among eukaryotes}

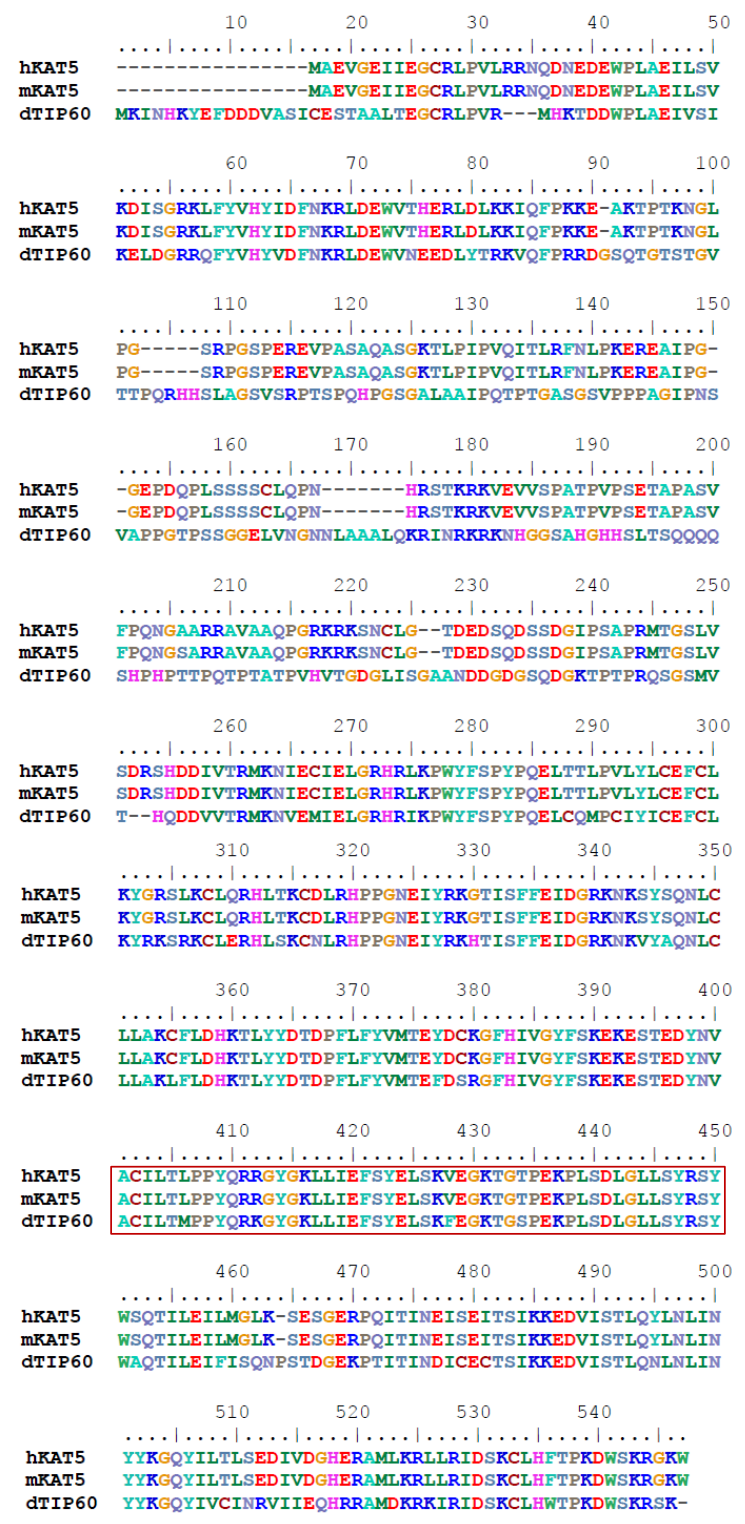

Figure 3.1: Multiple sequence alignment of TIP60/KAT5

Full length protein sequences aligned with BioEdit reveal a high degree of conservation among the three species human, mouse and fruit fly Drosophila. The catalytic region is highlighted inside red rectangle is also very well conserved across the species investigated. The carboxyl terminal is also well conserved which can be used as a strategy to generate antibodies against TIP60 across multiple species. 
TIP60/KAT5 is a well conserved protein among eukaryotes. TIP60/KAT5 belongs to MYST family of acetyltransferases (MOZ, YBF2, SAS2, TIP60) which are characterized by a 300 amino acid MYST domain containing a zinc finger and an acetylCoA binding motif. As shown in Figure 3.1 the orthologs of Drosophila TIP60, namely human and mouse KAT5 shows a high degree of conservation. Human and mouse orthologs are $100 \%$ near identical with one amino acid divergence at position 175, serine in mouse and alanine in human KAT5. The Drosophila TIP60 shares 89 to 90\% identify with human and mouse orthologs. The catalytic region or the acetyl Co-A binding motif is well conserved across three species. Drosophila TIP60 has highly conserved orthologs in all eukaryotes. The yeast ortholog Esal shares striking identity with other TIP60 orthologs in all eukaryotes, while in zebrafish there are two known orthologs as a result of gene duplication.

Three different isoforms of Tip60 transcripts have been reported (Sapountzi et al., 2006), however the predominant long isoform is well studied. Figure 3.2 summarizes the three known isoforms of Tip60 in Drosophila and the genomic location. RNA-seq signals for Tip60 from modENCODE project are presented in Figure 3.2 for different developmental stages and adult head under different conditions. It is very clear from this analysis that Tip60 is highly expressed during embryonic development. During larval transition Tip60 appears to be expressed at very low levels since the reads of the normalized signals are minimal. However, both females and males adult flies as well adult heads irrespective of mating status express Tip60 at detectable level. It is interesting to note that treatment with certain chemical s such as caffeine, cadmium and paraquat elevates the expression of Tip60. This provides an interesting start to investigate the effects of Tip60 in adult Drosophila circadian clock. 


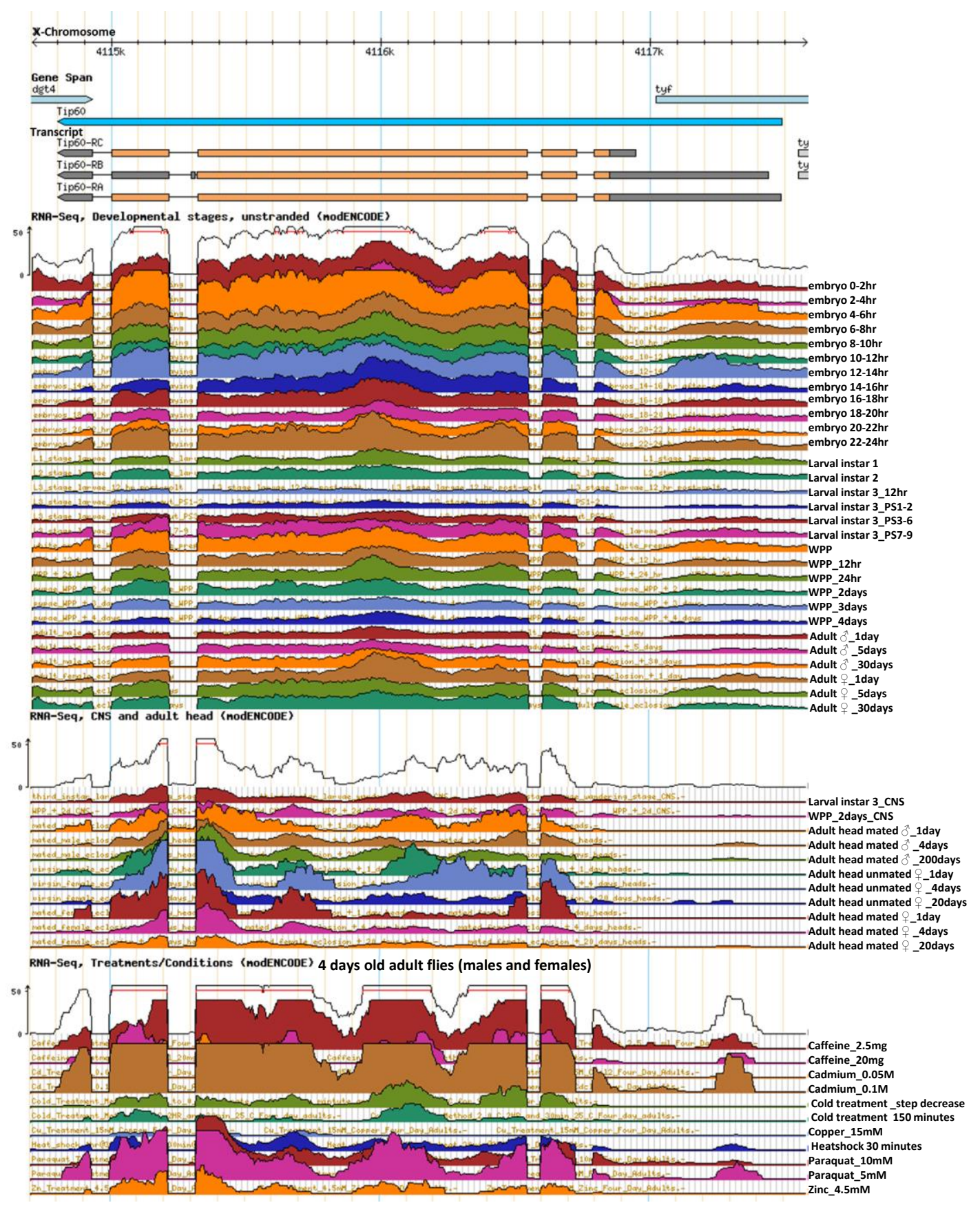

Figure 3.2: Transcriptome profile of Tip60 obtained from modENCODE data

Expression profiles for Tip60 exons from RNA-seq signal of different developmental stages and head tissue under different conditions and treatments were visualized using "RNA-seq by region tool" from Flybase (released on June 29, 2015). Adult tissues express less Tip60 in comparison to the early embryonic stages. Tip60 transcripts highly vary in adults upon treatment with chemical agents. 


\subsection{TIP60/KAT5 localizes in circadian pacemaker neurons}

Immunostaining using anti-dTIP60 antibody (Schirling et al., 2010) was performed to examine the endogenous expression of TIP60/KAT5 in the brain of adult Drosophila ( $w^{1118}$ control strain), that were entrained in LD cycle for five days. TIP60 staining was observed in the most regions of the brain (Figure $3.3 \mathrm{~B}, \mathrm{D}$ ). The circadian clock neurons express TIP60 endogenously as evident from co-staining with PDF (Figure $3.3 \mathrm{D}$ ). AntiPDF antibody was used to mark lateral ventral circadian pacemaker neurons (Figure 3.3 C).
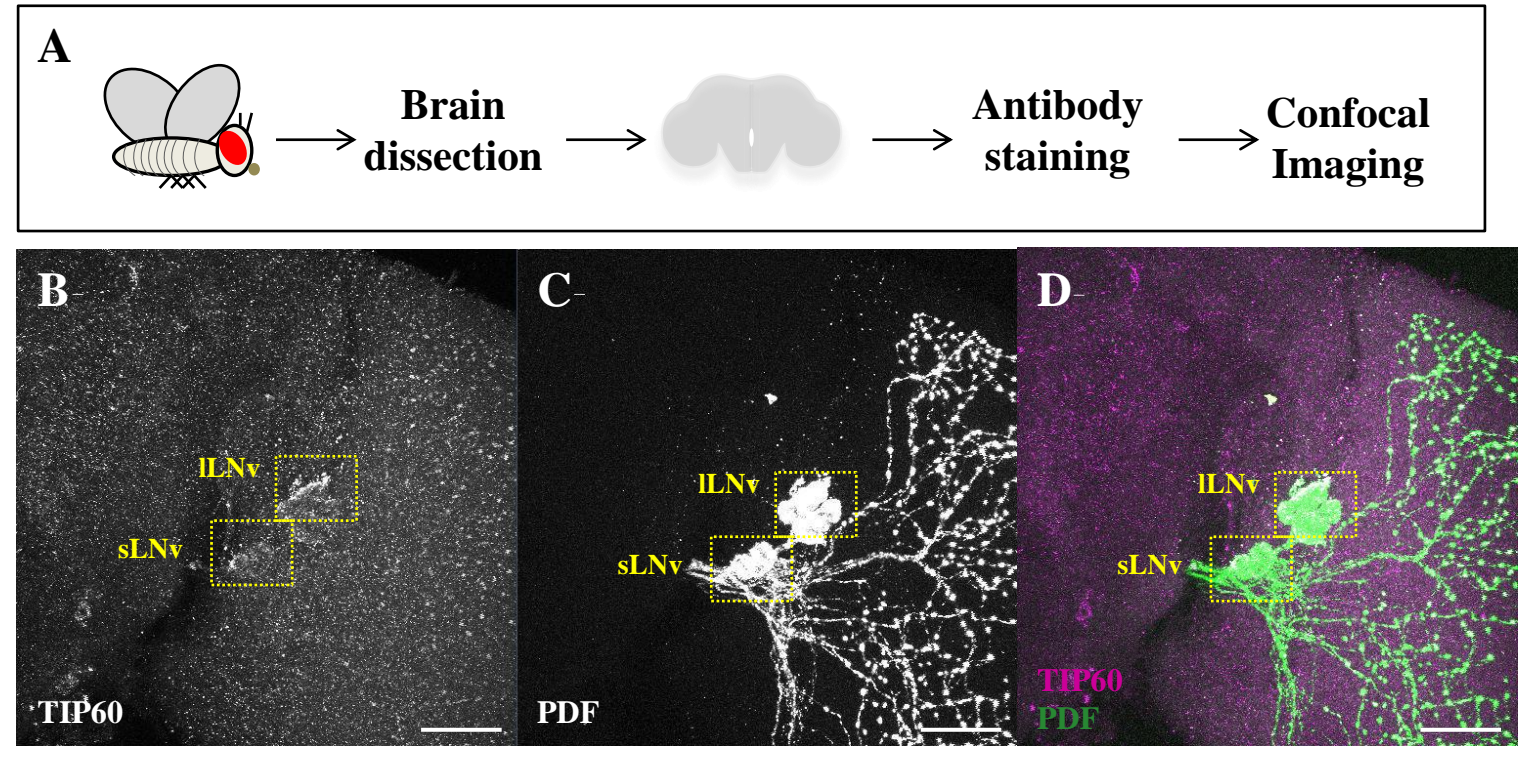

Figure 3.3: TIP60/KAT5 is expressed in clock neurons of adult Drosophila brain

(A) Schematic representation of immunostaining protocol. (B, C, D) Maximum intensity projection confocal micrographs from a representative adult brain of $w^{1118}$ strain entrained in LD cycle for 5 days, co-stained with anti-dTIP60 antibody and anti-PDF antibody. Merged signals from two channels are shown as indicated in $\mathbf{D}$, whereas signals from respective single channels are shown in $\mathbf{B}$ for dTIP60 and $\mathbf{C}$ for PDF. Cell bodies of lLNv- large lateroventral neurons, sLNv- small lateroventral neurons are indicated by yellow polygons. The scale bars represent 50 $\mu \mathrm{m}$. 


\subsection{Tools to manipulate TIP60/KAT5 levels in circadian pacemaker neurons}

Homozygous mutants of Tip60 in Drosophila are not viable and hence in order to investigate the specific effects of TIP60/KAT5 in the circadian pacemaker neurons, GAL4/ UAS binary expression genetic tool kit (Brand and Perrimon, 1993) was utilized. PdfGAL4 (Renn et al., 1999) was used to achieve targeted manipulation of TIP60 levels in the circadian pacemaker neurons. Since circadian pacemaker neurons express TIP60 endogenously (Figure 3.3) it would be a straightforward approach to utilize RNA interference to knockdown TIP60 in a spatially restricted manner. Overexpression of TIP60 ${ }^{\mathrm{E} 431 \mathrm{Q}}$, a mutation in the catalytic site that acts in a dominant negative fashion by UAS/GAL4 system had resulted in developmental defects (Lorbeck et al., 2011) therefore this would also be a valuable approach to manipulate TIP60 levels in the clock neurons.

Lateral ventral neurons specific knockdown of TIP60 did not show any viability defects during development at ambient temperature of $25^{\circ} \mathrm{C}$. Healthy adult flies were collected after emergence from pupal case and observed for visible morphological changes in comparison to their parental controls reared under similar conditions. Locomotor behavior of adult flies from this cohort was analyzed to rule out defects in the general health status due to targeted knockdown of Tip60 by RNAi and overexpression of TIP60 ${ }^{E 431 Q}$ mutant version (Table 3-1). Other usual behaviors such as flight, mating and egg-laying were also not affected (personal observation) indicating a normal sense of vision and olfaction in these flies.

\subsection{Validation of spatio-temporal TIP60 knockdown}

Knockdown of TIP60 in the pacemaker neurons using UAS/GAL4 system was detrimental to healthy adult survival. Hence, to restrict the knockdown only during adult specific stages, temperature sensitive TARGET system (McGuire et al., 2004) was used. Since lateroventral clock neurons constitute only a small subset of cells, it becomes technically challenging to assess the knockdown efficiency using qRT-PCR from a heterogeneous tissue such as fly heads. However, a pan-neuronal driver was used to determine the knockdown efficiency in a temporal fashion by rearing the flies of same 
genotype at permissive and restrictive temperatures during adulthood. (Figure $3.4 \mathrm{~A}, \mathrm{~B}$ ). When reared at permissive temperature of $18^{\circ} \mathrm{C}$, Tip60 mRNA did not significantly differ between the genotypes in the adult heads (Figure $3.4 \mathrm{C}$ ). However, at restrictive temperature of $30^{\circ} \mathrm{C}$ the knockdown was significantly observed in genotypes where RNAi for Tip60 was induced and $T I P 60^{E 431 Q}$ mutant version was overexpressed (Figure 3.4 D). Tukey's post hoc test revealed a significance of $p<0.001$ between pan-neuronal driven Tip $60^{R N A i}$ and TIP60 $0^{E 431 Q}$. The residual Tip60 levels $(\sim 50 \%)$ found in Tip $60^{\mathrm{RNAi}}$ could be from non-neuronal tissues such as cuticle present in the head. As expected, the overexpression of TIP $60^{E 431 Q}$ using Elav promoter showed three-fold higher levels of Tip60 transcript that was measured.

The next step was to assess if knockdown of TIP60 could be achieved using lateroventral clock neuron driver Pdf-Gal4. Adult flies that were allowed to develop at permissive temperature and then reared at restrictive temperature for 5 days were dissected and the brains were stained with anti-dTIP60 and anti-PDF antibodies. Knockdown of Tip60 using a $P d f$ promoter was determined to be specific for pacemaker neurons (Figure 3. 5). Since non-pacemaker neurons were also stained with dTIP60, it was possible to determine the knockdown in clock neurons within the same sample. Although Tukey's post hoc test revealed a significance of $p<0.05$ between the genotypes quantified for dTIP60 signal, there was an appreciable amount of TIP60 present in the clock neurons (Figure 3.5) either due to inefficient knockdown or sustained stability of TIP60 turnover following down regulation. Specificity of the anti-dTIP60 antibody is also compromised due to recognition of epitopes in the fixed tissue. When $T I P 60^{E 431 Q}$ is over expressed in clock neurons, the staining was observed all-around the cell (data not shown) suggesting the efficiency of overexpression by TARGET system. 

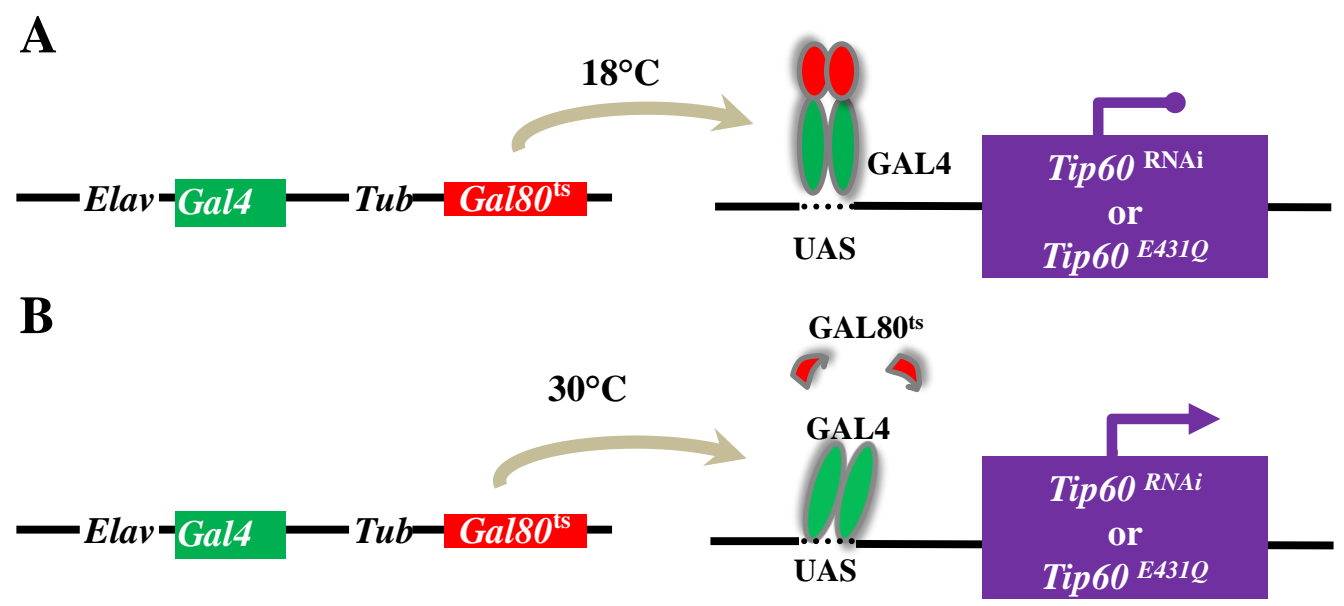

C

D
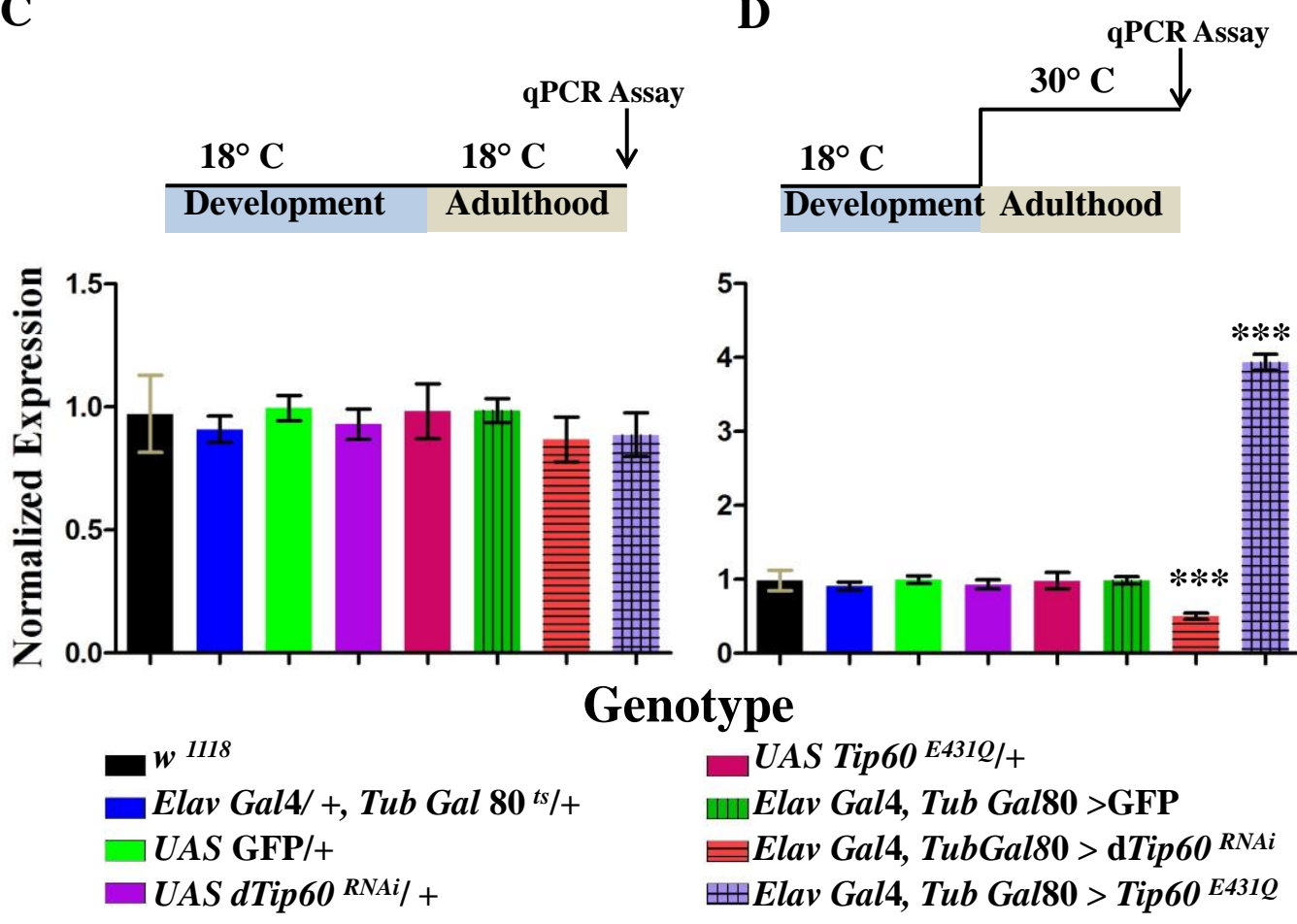

Figure 3.4: Validation of spatio-temporal Tip60 knockdown using TARGET

(A, B) Schematic representation of TARGET system to achieve spatial and temporal control of Tip60 knockdown or misexpression, using a pan-neuronal Elav driver. Cohorts were maintained at permissive temperature $\left(18^{\circ} \mathrm{C}\right)$ during development and later reared as adults for 5 days in permissive temperature $(\mathbf{C})$ as well as restrictive temperature (D) to monitor Tip60 mRNA levels in head extracts by quantitative RT-PCR. The error bars indicate $95 \%$ confidence interval of mean. $* * * p<0.001$ determined by one-way ANOVA, Tukey post-hoc test. 


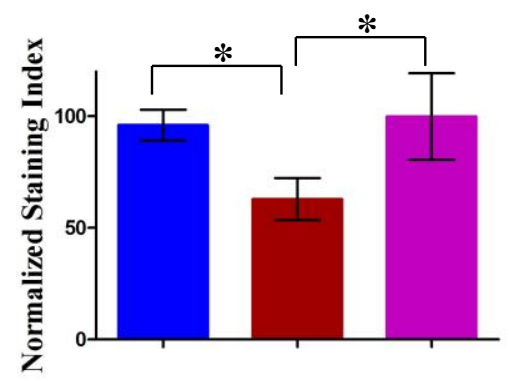

Pdf Gal4,Tub Gal $80>$ GFP
Pdf Gal4, Tub Gal $80>$ dTip60 ${ }^{\text {RNAi }}$
Pdf Gal4, Tub Gal $80>$ Tip60 $^{\text {E431Q }}$

Figure 3.5: Targeted knockdown and misexpression of dTIP60 in pacemaker neurons using temporal and regional gene expression targeting system

Staining intensity of dTIP60 in clock neurons labelled with PDF was quantitated and normalized to dTIP60 staining from the neighboring non-clock cells to assess the efficiency of knockdown. Error bars represent $95 \%$ confidence interval of means. Asterisk indicate $* p<0.05$ determined by one-way ANOVA, Bonferroni Post-hoc test.

\subsection{Adult lifespan is mediated by TIP60 in lateroventral clock neurons}

Although, knockdown of Tip60 in the circadian pacemaker neurons did not affect viability and certain aspects of healthy behavior in young adults, most flies from this cohort did not survive through a complete circadian protocol experiment spanning two weeks. Hence, a rigorous investigation of adult lifespan was performed at $25^{\circ} \mathrm{C}$. The data on adult life span revealed that flies with knockdown of TIP60 in lateral ventral neurons have a significant reduction in survival compared to their control counterparts (Figure 3.6 A, B). The average half-time of life span for flies that overexpress $T I P 60^{E 431 Q}$ mutant version in clock neurons is $16.83 \pm 0.4$ days (mean \pm SEM) (Figure 3.6 B) which is higher than for flies with knockdown of TIP60 in clock neurons $9.5 \pm 0.4$ days ; however KaplanMeier survival curve exposed the drop in adult survival from very early on. This data suggests that TIP60 is required in lateral ventral clock neurons to sustain a healthy ageing in Drosophila. Alternatively, reduction in adult lifespan due to knockdown of Tip60 might 
be an indirect effect arising from lack of proper axonal growth in these neurons as described in a previous study (Pirooznia et al., 2012).

A
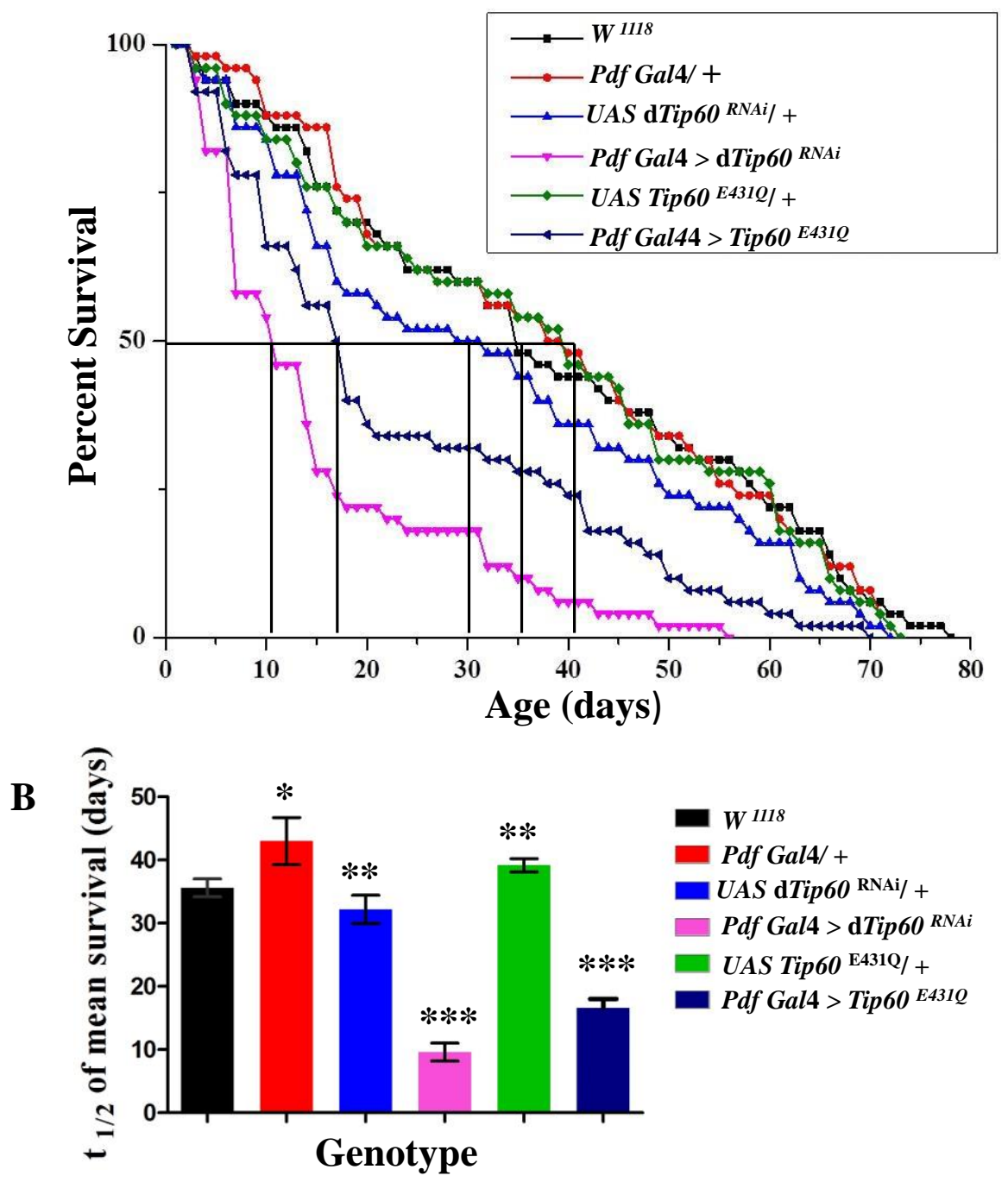

Figure 3.6: TIP60 in pacemaker clock neurons is required for healthy adult survival

(A) Kaplan-Meier survival plot of the indicated genotypes reveal TIP60 in pacemaker neurons is involved in mediating adult life span. A total of 100 flies were assayed for survival over time for each genotype at $25^{\circ} \mathrm{C} .50 \%$ of mean survival is indicated at the abscissa of the survivor curve bisected by a straight line. (B) Bar graph showing half time of mean survival in each genotype assayed. The error bars represent standard error of mean. * $p<0.05$. ** $p<0.01, * * * p<0.0001$ determined by one-way ANOVA, Tukey post-hoc test. 


\subsection{TIP60/KAT5 is required in adult pacemaker clock neurons for robust circadian behavioral rhythmicity}

Since TIP60 is expressed in adult circadian pacemaker neurons (Figure 3.3) and genetic tools to spatio-temporally knockdown are validated (Figure 3.4, 3.5) the role of TIP60 in regulating circadian rhythms was tested by locomotor behavior assay: a noninvasive strategy to assess the internal state of circadian clock. Flies exhibited rhythmic locomotor behavior when entrained to a 12:12 h LD cycle at $30^{\circ} \mathrm{C}$ (Figure 3.7A). Under constant free running conditions the animals with a knock down of TIP60 or overexpression of TIP60 ${ }^{\mathrm{E} 431 \mathrm{Q}}$ in clock neurons displayed arrhythmic to aberrant complex rhythm (Figure 3.7 $\mathrm{C}$ and Table 3.1) compared to the controls. The rhythm in constant dark (DD) conditions was determined both by $\chi$-square (Figure $3.7 \mathrm{~B}$ ) and Lomb-Scargle periodogram to rule out biases arising due to sinusoidal wave fitting. Free running period of animals that did not match circadian periodicities ( 22 to $26 \mathrm{~h}$ ) were classified as complex rhythm (Figure 3.7 C, Table 3.1). Up to $60 \%$ of animals were rendered arrhythmic due to TIP60 ${ }^{E 431 Q}$ misexpression in clock neurons at restrictive temperature (Figure $3.7 \mathrm{C}$ ). Power of rhythmicity was measured for the genotypes tested and the lower power values for the mutants reflect a lesser robustness of rhythms observed (Figure 3.7 D). Three independent responder strains for RNAi and two strains for $T I P 60^{E 431 Q}$ were tested.

Overexpression of $T I P 60^{E 431 Q}$ in clock neurons reflected a stronger behavioral phenotype in comparison to knockdown of TIP60 (Table 3-1), suggesting either a lesser efficiency of RNAi responder strains or a dominant negative effect of the catalytic mutant overexpression. Complex rhythm of period lengthening due to knockdown of TIP60 was consistent with independent responder RNAi strains (Table 3-1) and could be attributed to partial penetrance due to exogenous manipulation of TIP60. The increased percentage of arrhythmic behavior is clearly an adult specific loss of TIP60, since rearing adult animals at permissive temperature throughout locomotor assay does not cause any significant behavioral changes (Table 3-1). This indicates a strong requirement of TIP60/KAT5 in lateroventral pacemaker neurons during adulthood for robust functioning of the circadian clock. 
Table 3-1: Rhythmicity and period length of TIP60 expression in clock neurons investigated in constant darkness (DD) according to $\chi^{2}$-periodogram analysis

\begin{tabular}{|c|c|c|c|c|c|}
\hline Genotype & $n^{1}$ & AR \% ${ }^{2}$ & $\mathrm{CR}^{3}{ }^{3}$ & SR \% ${ }^{4}$ & 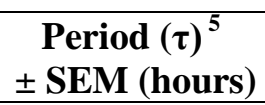 \\
\hline \multicolumn{6}{|l|}{$\mathrm{DD}$ at $25^{\circ} \mathrm{C}$} \\
\hline$w^{1118}$ & 32 & 0 & 3 & 97 & $23.7 \pm 0.3$ \\
\hline$P d f-G a l 4 /+$ & 27 & 0 & 4 & 96 & $23.8 \pm 0.5$ \\
\hline$U A S-\mathrm{d} T i p 60^{R N A i} /+$ \#A & 18 & 5 & 12 & 83 & $24.5 \pm 0.2$ \\
\hline$U A S-\mathrm{d} T i p 60^{R N A i} /+$ \#B & 21 & 0 & 0 & 100 & $23.5 \pm 0.7$ \\
\hline$U A S-\mathrm{d} T i p 60^{R N A i} /+\# \mathrm{C}$ & 28 & 0 & 2 & 98 & $23.8 \pm 0.4$ \\
\hline$P d f$-Gal4 >dTip $60^{R N A i} \# \mathrm{~A}$ & 12 & 42 & 25 & 33 & $27.2 \pm 0.2$ \\
\hline$P d f-G a l 4>\mathrm{d}$ Tip $60^{R N A i} \# \mathrm{~B}$ & 17 & 58 & 24 & 18 & $25.9 \pm 0.1$ \\
\hline Pdf-Gal4 >dTip $60^{R N A \mathrm{i}} \# \mathrm{C}$ & 9 & 66 & 22 & 11 & $25.7 \pm 0.2$ \\
\hline$U A S-T i p 60^{E 43 I Q} /+$ \#A & 26 & 4 & 20 & 76 & $24.5 \pm 0.2$ \\
\hline$U A S-T i p 60^{E 431 Q} /+$ \#B & 31 & 0 & 10 & 90 & $23.8 \pm 0.6$ \\
\hline Pdf-Gal4 >Tip $60^{E 431 Q}$ \#A & 19 & 62 & 28 & 10 & $24.6 \pm 0.1$ \\
\hline Pdf-Gal4 >Tip $60^{E 431 Q}$ \#B & 13 & 73 & 21 & 6 & $25.6 \pm 0.3$ \\
\hline \multicolumn{6}{|l|}{ DD at $30^{\circ} \mathrm{C}$} \\
\hline$w^{1118}$ & 15 & 0 & 0 & 100 & $23.4 \pm 0.2$ \\
\hline Pdf-Gal4; Tub-Gal80 ${ }^{\mathrm{ts}} /+$ & 42 & 2 & 10 & 88 & $23.6 \pm 0.5$ \\
\hline$U A S-\mathrm{d} T i p 60^{R N A i} /+$ \#A & 31 & 6 & 16 & 78 & $23.7 \pm 0.3$ \\
\hline$U A S-\mathrm{d} T i p 60^{R N A i} /+$ \#B & 29 & 0 & 10 & 90 & $23.2 \pm 0.5$ \\
\hline$U A S-\mathrm{d} T i p 60^{R N A i} /+\quad \# \mathrm{C}$ & 22 & 9 & 18 & 73 & $23.5 \pm 0.2$ \\
\hline$P d f$-Gal4;Tub-Gal80>dTip $60^{R N A i} \# \mathrm{~A}$ & 26 & 65 & 12 & 23 & $26.4 \pm 0.8$ \\
\hline$P d f$-Gal4;Tub-Gal80>dTip $60^{R N A i} \# \mathrm{~B}$ & 30 & 60 & 20 & 20 & $25.3 \pm 0.4$ \\
\hline$P d f$-Gal4;Tub-Gal80>dTip $60^{R N A i} \# \mathrm{C}$ & 25 & 56 & 24 & 20 & $24.7 \pm 0.6$ \\
\hline$U A S-T i p 60^{E 431 Q} /+\# \mathrm{~A}$ & 36 & 8 & 17 & 75 & $24.3 \pm 0.4$ \\
\hline$U A S-T i p 60^{E 431 Q} /+$ \#B & 48 & 6 & 12 & 82 & $23.7 \pm 0.6$ \\
\hline$P d f$-Gal4;Tub-Gal80>Tip $60^{E 431 Q}$ \#A & 19 & 58 & 24 & 18 & $25.3 \pm 0.7$ \\
\hline Pdf-Gal4;Tub-Gal80>Tip60 ${ }^{E 431 Q}$ \#B & 21 & 62 & 28 & 10 & $25.8 \pm 0.1$ \\
\hline \multicolumn{6}{|l|}{$\mathrm{DD}$ at $18^{\circ} \mathrm{C}$} \\
\hline$w^{1118}$ & 12 & 0 & 0 & 100 & $23.8 \pm 0.3$ \\
\hline Pdf-Gal4; Tub-Gal80 ${ }^{\mathrm{ts}} /+$ & 16 & 0 & 0 & 100 & $23.5 \pm 0.7$ \\
\hline$U A S-\mathrm{d} T i p 60^{R N A i} /+$ \#A & 14 & 0 & 12 & 88 & $23.9 \pm 0.1$ \\
\hline$U A S-\mathrm{d} T i p 60^{R N A i} /+$ \#B & 16 & 0 & 0 & 100 & $23.5 \pm 0.3$ \\
\hline$U A S-\mathrm{d} T i p 60^{R N A i} /+\# \mathrm{C}$ & 15 & 0 & 6 & 94 & $23.7 \pm 0.7$ \\
\hline$P d f$-Gal4;Tub-Gal80>dTip $60^{R N A i} \# \mathrm{~A}$ & 15 & 6 & 19 & 75 & $23.7 \pm 0.4$ \\
\hline$P d f$-Gal4;Tub-Gal80>dTip $60^{R N A i} \# \mathrm{~B}$ & 16 & 0 & 13 & 87 & $23.9 \pm 0.7$ \\
\hline$P d f$-Gal4;Tub-Gal80>dTip $60^{R N A i} \# \mathrm{C}$ & 14 & 0 & 21 & 79 & $24.0 \pm 0.5$ \\
\hline$U A S-T i p 60^{E 431 Q} /+$ \#A & 10 & 0 & 40 & 60 & $24.7 \pm 0.3$ \\
\hline$U A S-T i p 60^{E 431 Q} /+$ \#B & 16 & 0 & 15 & 85 & $24.1 \pm 0.9$ \\
\hline$P d f$-Gal4;Tub-Gal80>Tip $60^{E 431 Q}$ \#A & 15 & 13 & 26 & 61 & $23.8 \pm 0.3$ \\
\hline$P d f$-Gal4;Tub-Gal80>Tip $60^{\mathrm{E} 431 Q}$ \#B & 13 & 0 & 19 & 81 & $24.2 \pm 0.6$ \\
\hline
\end{tabular}

${ }^{1} \boldsymbol{n}$ indicates the number of flies tested in each genotype that survived locomotor recordings.

${ }^{2}$ Arrhythmic, ${ }^{3}$ Complex rhythms, ${ }^{4}$ StronglyRhythmic in circadian range.

${ }^{5}$ Period $(\tau)$ in DD is determined by $\chi^{2}$-periodogram analysis. 
A

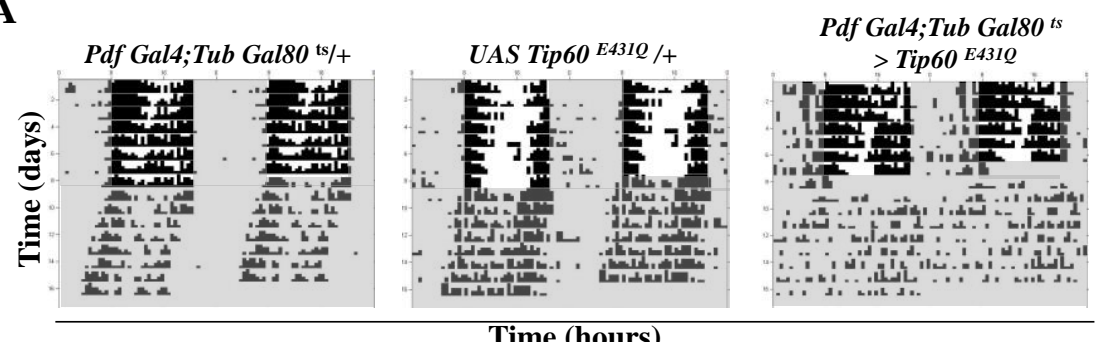

B
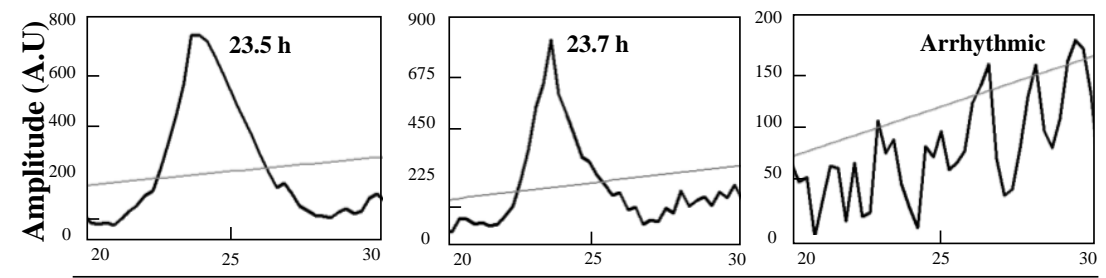

Period (hours)
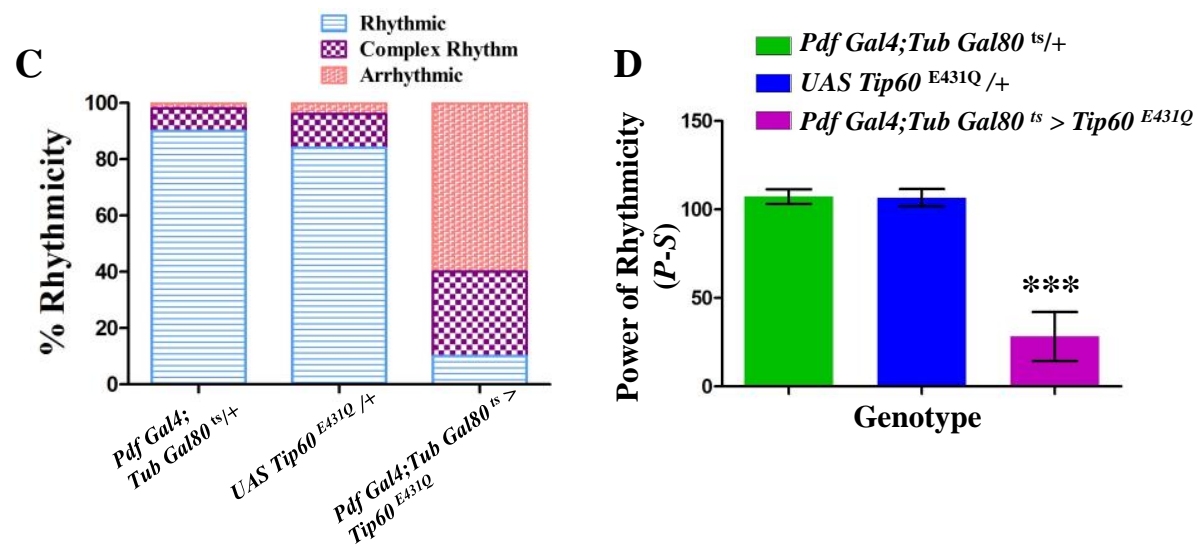

Figure 3.7:TIP60/KAT5 mutants have aberrant free running rhythms

(A) Double plotted actograms of representative individual flies, showing normalized activity as vertical black bars against the days in $\mathrm{Y}$ axis and time of the day in $\mathrm{X}$ axis. The grey background regions represent dark conditions while the white regions indicate the laboratory light phase of the experiment. In general, the mutant flies (right panel) have an arrhythmic locomotor activity patterns in the free running condition whereas the wild type controls (left and middle panels) restrict their activity in a rhythmic fashion when assayed at restrictive temperature of $30^{\circ} \mathrm{C}$. $\chi$-square periodogram of representative animals showing the significant periods determined during free running conditions. (C) Distribution of percentage rhythmic animals as classified by periodogram analysis. Greater percentage of arrhythmic individuals is found in mutant cohorts in addition to complex rhythms. (D) Power of rhythmicity (as measured by power $(P)$ - significance $(S)$ values) under constant free running conditions of the genotypes tested. Data represent mean \pm SEM. $* * * p<0.0001$ determined by one-way ANOVA, Dunnet post-hoc test. 


\subsection{Overexpression of $T I P 60^{E 431 Q}$ in adult clock neurons does not impair PDF neuropeptide expression and the morphology of pacemaker neuronal network}

Although, temporal knockdown of Tip60 and overexpression of TIP60 ${ }^{E 431 Q}$ caused significant reduction in rhythmic locomotor under constant darkness, it is not clear if such a drastic change in behavior is due to impaired neuronal connectivity or the disturbance of core molecular circadian clock per se. Hence, the adult brains of flies overexpressing $T I P 60^{E 431 Q}$ were examined for morphology of pacemaker network using neuropeptide PDF as a marker. Flies with overexpression of $T I P 60^{E 431 Q}$ in pacemaker neurons pose an interesting case, with a strong behavioral phenotype in order to tease out the deficiency arising from core clock with that of general cellular effects mediated by TIP60/KAT5 in clock neurons. Flies harboring a knockdown of Tip60 by RNAi were excluded for further analysis of clock function, since the partially penetrant phenotype would mask any difference observed at population level.

Neuropeptide PDF immunoreactivity was observed in control and mutant groups enabling the examination of clock neuron network morphology (Figure 3.8). Small and large ventral lateral neurons along with dorsal projections and protocerebral tract were present irrespective of temperature treatment and genotype (Figure $3.8 \mathrm{C}$ ). It is highly unlikely; these neurons would have failed to form appropriate connections, since the misexpression was induced post emergence only during adult stages. Irrespective of this speculation, the mutant animals clearly show anticipation before lights on during entrainment (Figure 3.7A) suggesting that ventral lateral neurons are integrated to synchronize with the environmental cues. Given that, the staining pattern of anti-PDF antibody in the mutants was completely normal (Figure 3.8), the likely explanation of arrhythmic locomotor behavior (Figure 3.7) could be certainly due to a malfunction of the core clock machinery. Although, signal intensity of PDF immunoreactivity was generally increased when reared at $30^{\circ} \mathrm{C}$, this did not affect the morphology of axonal terminals in dorsal horn projections (DP) or medullary projections (MP). Protocerebral tract was also clearly visible in every case suggesting a sustainment of hemispherical connectivity. PDF signaling in the nerve terminals of medullary projection and dorsal projections did not show any change due to misexpression of TIP60 ${ }^{E 431 Q}$ (Figure 3.8 C). 

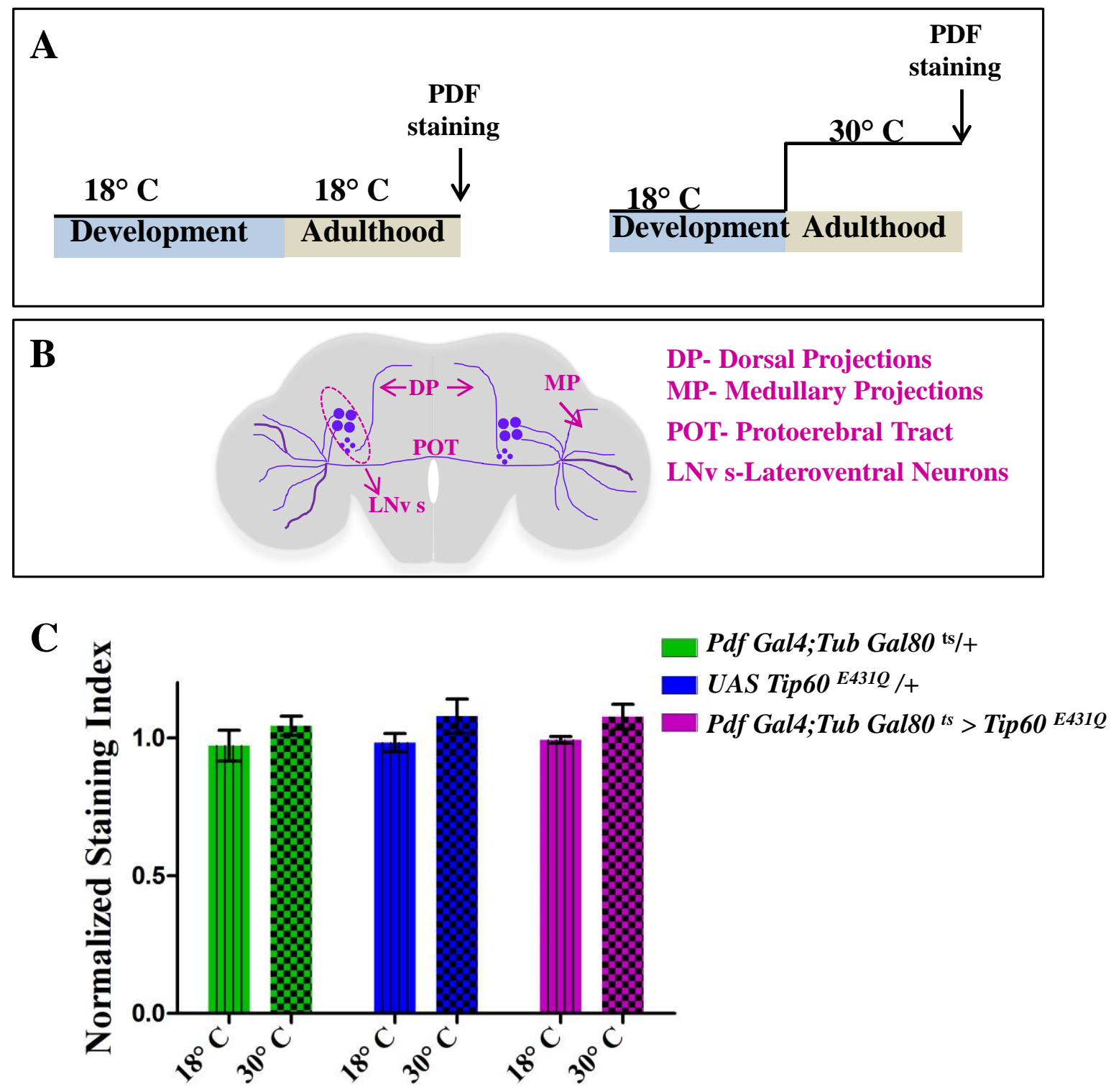

Figure 3.8: Spatiotemporal expression of $T I P 60^{E 431 Q}$ in pacemaker clock neurons does not disrupt PDF neuropeptide expression.

(A) Schematic representation of the experimental design used to achieve spatiotemporal pattern ofTIP6 $0{ }^{\mathrm{E} 431 \mathrm{Q}}$ misexpression in clock neurons. (B) Schematic representation of PDF immunoreactivity anatomy in the brain. (C) Quantification of PDF levels in axon termini of the medullary projections. 20 adult brains were examined for every genotype and experimental condition. Error bars represent standard error of mean. Gross morphology of ventral lateral neurons observed at Zeitgeber Time 05 (ZT5) in all cases was not affected in the genotypes examined. 


\subsection{Expression of core clock controlled genes are modulated by TIP60}

In order to test if the arrhythmic locomotor behavior observed in flies misexpressing $T I P 60 E^{431 Q}$ in clock neurons is arising due to a molecular deficiency in the core clock machinery, gene expression analysis of core clock genes and clock controlled genes was carried out in whole heads. The flies were raised at permissive temperature during development and then switched to restrictive temperature as adults allowing entrainment at LD cycles for 5 days. On the second day of DD, flies were collected over different time points and the transcripts of clock controlled genes were measured using a quantitative real time PCR. No significant differences in the mRNA levels of the activators Clock $\left(\mathrm{F}_{(2,18)}=\right.$ 8.96, $p=0.814)$ and Cycle $\left(\mathrm{F}_{(2,18)}=1.37, p=0.63\right)$ were observed between the controls and mutant groups (Figure 3.9). Since the activators themselves are not regulated by E-box, it is not a surprise that these transcripts do not change significantly as revealed by Two-way ANOVA, Bonnferroni post hoc test. However the key transcripts of clock controlled genes and the auxiliary loop genes that are strongly under the control of E-boxes were rhythmic in the control groups under constant conditions while the mutants show strongly damped oscillations (Figure 3.9). Two-way ANOVA, Bonnferroni post hoc test revealed a highly significant effect for Period $\left(\mathrm{F}_{(2,18)}=17.15, p<0.01\right)$, Timeless $\left(\mathrm{F}_{(2,18)}=58.43, p<0.001\right)$, Vrille $\left(\mathrm{F}_{(2,18)}=68.14, p<0.0001\right)$ and Clockwork orange $\left(\mathrm{F}_{(2,18)}=59.27, p<0.0001\right)$ at peak of their expression over circadian time.

The head of Drosophila, however is a very heterogeneous tissue consisting of neurons, glia, fat tissue, cuticle and ommattidia bathed in hemolymph and tracheal connections. Most circadian signals from the head are contributed by ommatidia (Emery, 2007), reflecting the synchrony between tissues towards the pace set by central clock neurons. Hence assay of transcripts from the whole head may fail to reflect precise molecular underpinning arising only from a manipulation of a subset of neurons in the lateroventral region of the brain. Immunostaining was performed in another cohort of flies, raised under similar conditions

using anti-PER antibody to reveal molecular oscillations of the clock and anti-PDF antibody to label the pacemaker neurons. Since PER is expressed rhythmically in clock neurons that are not positive for PDF (lateral dorsal neurons and dorsal neurons), this assay would enable 
to assess if the synchrony of molecular rhythms in these subset of cells are preserved upon manipulation of lateroventral clock neurons. PER signals were measured over circadian cycle after normalization to surrounding regions of same area, within the same preparation to avoid bias in quantification. PER was found to be expressed with a strong rhythmic pattern in the clock neurons of control groups (Figure 3.10). The mutants display a damped PER expression (Figure 3.10, right panel) both in the PDF positive lateroventral neurons (Figure $3.10 \mathrm{~B})\left(\mathrm{F}_{(3,24)}=136.47, p<0.0001\right)$ and lateral dorsal neurons (Figure $\left.3.10 \mathrm{C}\right)\left(\mathrm{F}_{(3 \text {, }}\right.$ 24) $=230.07, p<0.0001$ ) revealing an extremely significant effect by Two-way ANOVA, Bonferroni post hoc test.

Stronger reduction of PER in LNds while manipulating LNvs exogenously suggest the role of pace communication between clock neuron groups. Most clock neurons that are negative for PDF, express receptor for PDF (Im and Taghert, 2010) and hence are strongly coupled. Misexpression of TIP60 in LNvs, do not affect the coupling between other groups of clock neurons and hence the pace is effectively communicated to cells coupled through molecular signatures (Figure $3.10 \mathrm{~B}, \mathrm{C}$ ). This is perhaps the reason for observing an extremely significant effect of clock controlled genes even in heterogeneous whole heads (Figure 3.9). The dampening of peak levels of clock controlled genes in mutants reveals a defect in the dynamic process of establishing temporal transcriptional signatures to the tissue by the core clock. This is in consistent agreement with the observed behavioral rhythmicity. Taken together, the data on transcript analysis in heads and PER immunostaining in clock neurons over circadian cycle clearly reveal that the core molecular machinery of clock is perturbed by TIP60 misexpression in lateroventral clock neurons. 


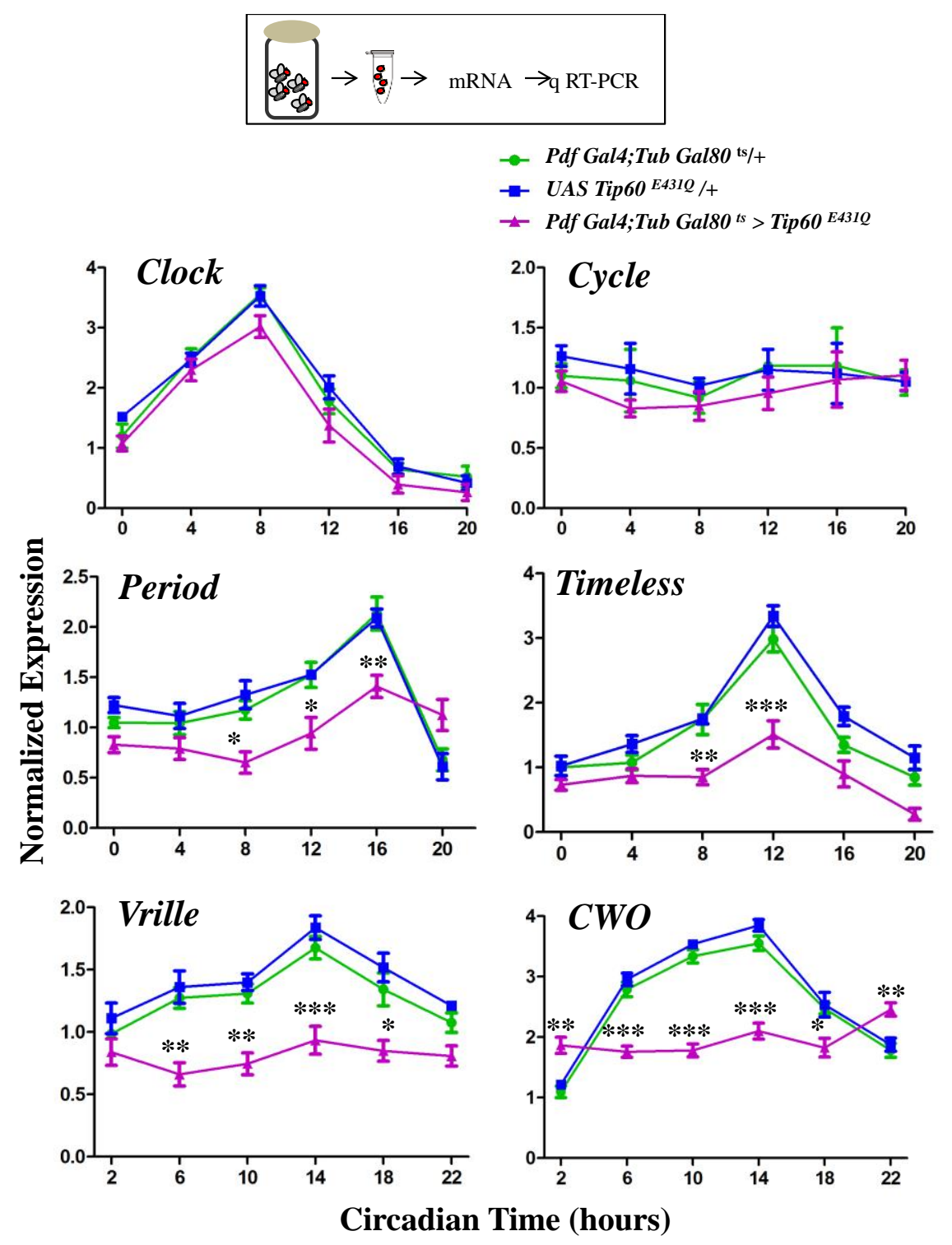

Figure 3.9: Transcriptional effects of $\mathrm{TIP}^{\mathrm{E} 431 \mathrm{Q}}$ misexpression in the circadian clock

Quantitative RT-PCR measurements of Clock (upper left panel), Cycle (upper right panel), Period (middle left panel), Timeless (middle right panel), Vrille (lower left panel) and Clock Work Orange (lower right panel)mRNA levels in head extracts of flies collected on the second day of free running. The flies were entrained for 5 days at $30^{\circ} \mathrm{C}$ and then released into constant darkness (DD) for 36 hours before the first time point of collection. CT0 corresponds to $36 \mathrm{~h}$ after the lights-off. Non-oscillating Rpl32 mRNA was used as internal control for normalization. Average values from four independent biological replicates are normalized to the mean of first time point control values set to 1. Error bars represent standard error of mean. Two-way ANOVA, Bonferroni multiple comparisons was used to determine $* p<0.05$, ** $p<0.01$, *** $p<0.0001$. 


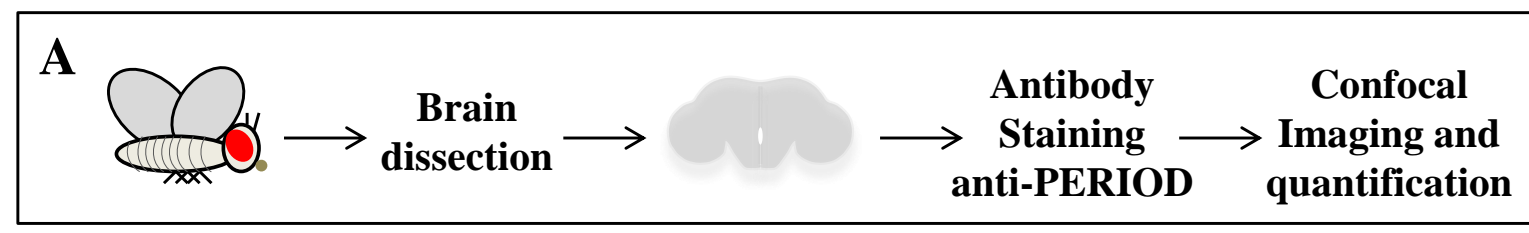

$\rightarrow$ Pdf Gal4;Tub Gal80 ts/+
$\rightarrow$ UAS Tip60 E431Q /+
$\leftarrow$ Pdf Gal4;Tub Gal80 ${ }^{\text {ss }}>$ Tip60 E431Q

B

C
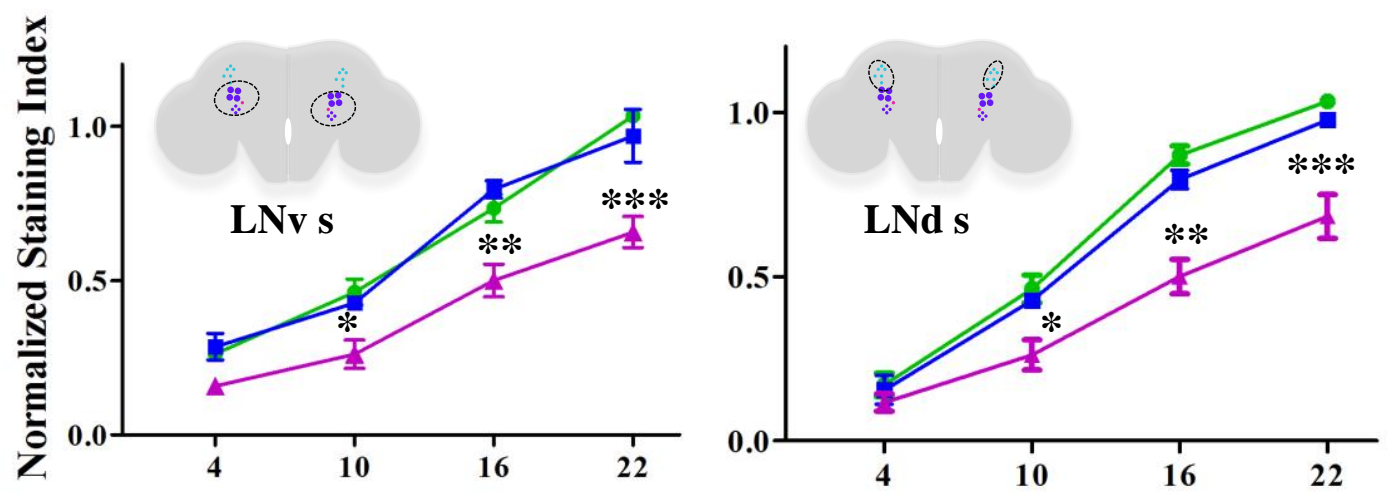

Circadian Time (hours)

Figure 3.10: TIP60 $^{E 431 Q}$ overexpression strongly dampens PER oscillations in clock neurons Animals were entrained in 12:12 h LD cycles at $30^{\circ} \mathrm{C}$ for five days and then released to constant darkness (DD) after the last lights-off of LD cycle. CT4 corresponds to 40 hours after lights-off on the second day of DD. (A) Schematic representation of the experimental protocol. Quantification was performed on confocal images of lateral clock neurons obtained at each time point by co-staining with anti-PDF and anti-PER antibodies (LNd- dorsal LN, LNv- ventral LN). (B) Quantification of PER signal in LNv s and (C) LNd, the relative location is depicted in the inset schematic cartoon. Data were collected from minimum of 20 brain hemispheres. Error bars represent standard error of mean. ${ }^{*} p<0.05, * * p<0.01$, *** $p<0.0001$ was determined by twoway ANOVA, Bonferroni post-hoc test. 


\subsection{Tip60 mRNA does not oscillate}

Expression profile of Tip60 in the wild type fly head was measured by quantitative real time PCR to determine if Tip60 mRNA is rhythmic in tissue. Gene transcript of Tip60 assayed as a function of circadian time did not show significant effect in fly heads (Figure 3.11 A). However Timeless, a strong clock controlled gene was found to be oscillating with a significant peak at CT $12\left(\mathrm{~F}_{(5,24)}=10.49, p<0.0001\right)$. Tip60 transcript was also assayed in heads of different clock mutants to tease out the regulation of TIP60 by clock. Remarkable differences in Tip60 mRNA levels were not observed in any of the clock mutants compared to wild type control at CT 12 (Figure 3.11 B). Tip60 transcripts are however expressed at very low abundance in adult heads due to the high cycle threshold values obtained consistently in independent experiments at different temperature regimen (personal observation, also in Figure 3.4 C, D).

Enzymes such as acetyltransferase TIP60 are perhaps not required in high concentrations in the cell and hence such an effect was observed. Hence it is highly unlikely that clock mutants would lack normal levels of Tip60 expression in adult tissues which could lead to several compromises in health of the cells. It should be noted that most post mitotic neurons in adult brain stain for TIP60 (Figure 3.3). Expression of Tip60 is found elevated during development and it is necessary for controlling gene expression in the nervous system (Lorbeck et al., 2011). TIP60 was also found not be oscillating on day time dependent manner in a previously published report (Pirooznia et al., 2012). This observation further strengthens the idea that TIP60 could be regulated independent of the clock and E-boxes at transcriptional level to carry forward variety of cellular and metabolic processes. Apart from the involvement of TIP60 in transcriptional control, it is known to play a strong role in DNA damage repair (Ikura et al., 2000; Kusch et al., 2004). Although TIP60 possess intrinsic acetyltransferase activity, it does not function in isolation in vivo but rather is found as a catalytic subunit of Nu4A multimeric protein complex (Sapountzi et al., 2006). Investigation of individual subunits in this multimeric protein complex in the context of circadian clock would be interesting since TIP60 forms a transient interaction with appropriate target proteins depending on the cellular context it participates. 
A

\begin{tabular}{cccc}
$4,009,000$ & $4,010,000$ & X chromosome & $4,011,000$ \\
\hline$\uparrow$ & Tip60 & $\uparrow$ \\
& & 105 bp in UTR
\end{tabular}

B

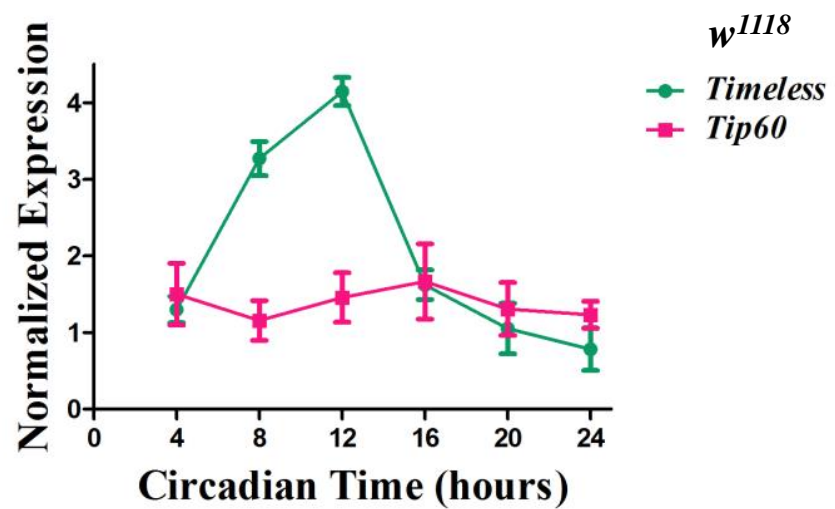

C

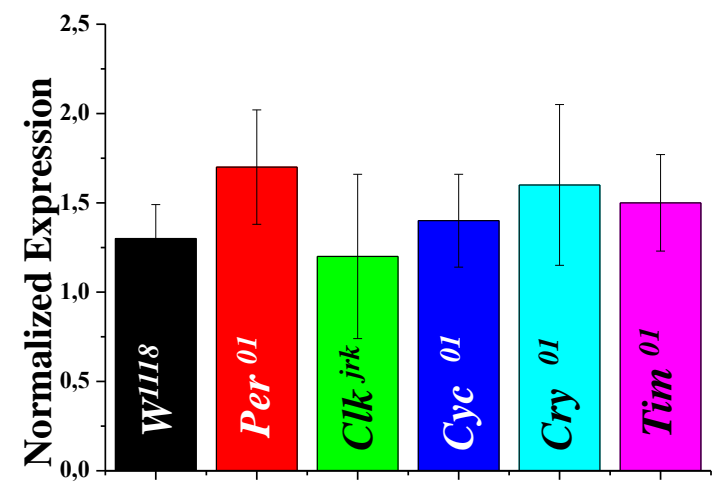

Genotype

Figure 3.11: Tip60 mRNA is not controlled by the circadian clock

(A) A schematic of the genomic region of Tip60showing the different regions which were chosen for mRNA analysis by quantitative real time PCR. The sizes of the amplicons are denoted for two different regions. (B) Gene expression analysis as a function of circadian time for Timeless and Tip60 mRNA in fly heads. $w^{1118}$ control flies were entrained for 5 days at $25^{\circ} \mathrm{C}$ and then released into constant darkness (DD) for 40 hours before the first time point of collection. Rpl32 mRNA was used as internal control for normalization. (C) mRNA levels of Tip60 in circadian clock mutants measured after 48 hours in DD (CT12). Clock mutants do not show any significant change in Tip60 at CT 12. Error bars in (B) and (C) represent SEM of four independent biological samples. 


\subsection{Subunits of Nu4A multimeric protein complex that interact with TIP60 contribute to robustness in behavioral rhythm.}

Since TIP60 is known to exert its activity in concert with several subunits of a multimeric protein complex (Sapountzi et al., 2006), locomotor behavioral assay was employed to screen candidates of the multimeric complex that would lead to circadian arrhythmic behavior. RNAi was used to individually knock down the expression of each of the subunits (Figure $3.12 \mathrm{~A}$ ) in lateroventral neurons during adult stages. Following entrainment under standard $\mathrm{LD}$ conditions at $30^{\circ} \mathrm{C}$, flies in which individual subunits of the Nu4A complex were knocked down by RNAi showed a period lengthening compared to their parental controls (Table 3-2, Figure 3.12 B). Analysis revealed that knockdown of p400 (Domino) appears to strongly mimic the arrhythmic phenotype and longer period reminiscent of Tip60 knockdown (Figure $3.12 \mathrm{~B}, \mathrm{C}$ ). Rhythm disturbances were observed among flies with knockdown of $E(P c)$, Ing3, dEAF6 which are subunit members of the NuA4 multimeric complex (Figure $3.12 \mathrm{~B}, \mathrm{C})$. This suggests that subunits of Nu4A complex is necessary for maintaining a normal circadian period and knockdown of one of the critical subunits that interact with TIP60 to exert catalytic function may be sufficient to impair the catalytic function in circadian context.

Table 3-2: Period length at $30^{\circ} \mathrm{C}$ after knockdown of $\mathrm{Nu} 4 \mathrm{~A}$ complex individual subunits

\begin{tabular}{|c|c|c|c|}
\hline Genotype & $n$ & Rhythmic \% & $\begin{array}{c}\text { Period ( }(\tau) \pm \text { SEM } \\
\text { (hours) }\end{array}$ \\
\hline Pdf-Gal4; Tub-Gal80 /s + & 27 & 96 & $23.6 \pm 0.2$ \\
\hline UAS-dTip $60^{R N A i}++$ \#A & 24 & 88 & $24.0 \pm 0.9$ \\
\hline Pdf-Gal4;Tub-Gal80>dTip60 ${ }^{R N A i} \# \mathrm{~A}$ & 29 & 18 & $26.8 \pm 0.5$ \\
\hline UAS-dom $m^{R N A i} /+$ & 32 & 97 & $23.8 \pm 0.1$ \\
\hline Pdf-Gal4;Tub-Gal80>dom ${ }^{R N A i}$ & 25 & 22 & $26.3 \pm 0.7$ \\
\hline$U A S-E(p c)^{R N A i} /+$ & 30 & 92 & $23.7 \pm 0.2$ \\
\hline Pdf-Gal4;Tub-Gal80> E(pc $)^{R N A i}$ & 27 & 76 & $24.8 \pm 0.1$ \\
\hline$U A S$-Ing $3^{R N A i} /+$ & 16 & 88 & $23.8 \pm 0.2$ \\
\hline Pdf-Gal4;Tub-Gal80>Ing $3^{R N A i}$ & 12 & 34 & $24.2 \pm 0.4$ \\
\hline$U A S-\mathrm{d} E a f^{R N A i} /+$ & 15 & 100 & $23.9 \pm 0.1$ \\
\hline Pdf-Gal4;Tub-Gal80>dEaf $f^{R N A i}$ & 11 & 82 & $23.9 \pm 0.3$ \\
\hline
\end{tabular}

Period $(\tau)$ in DD is determined by $\chi^{2}$-periodogram analysis 
A

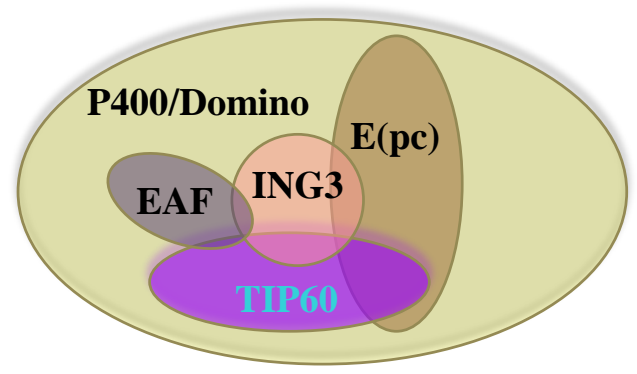

B
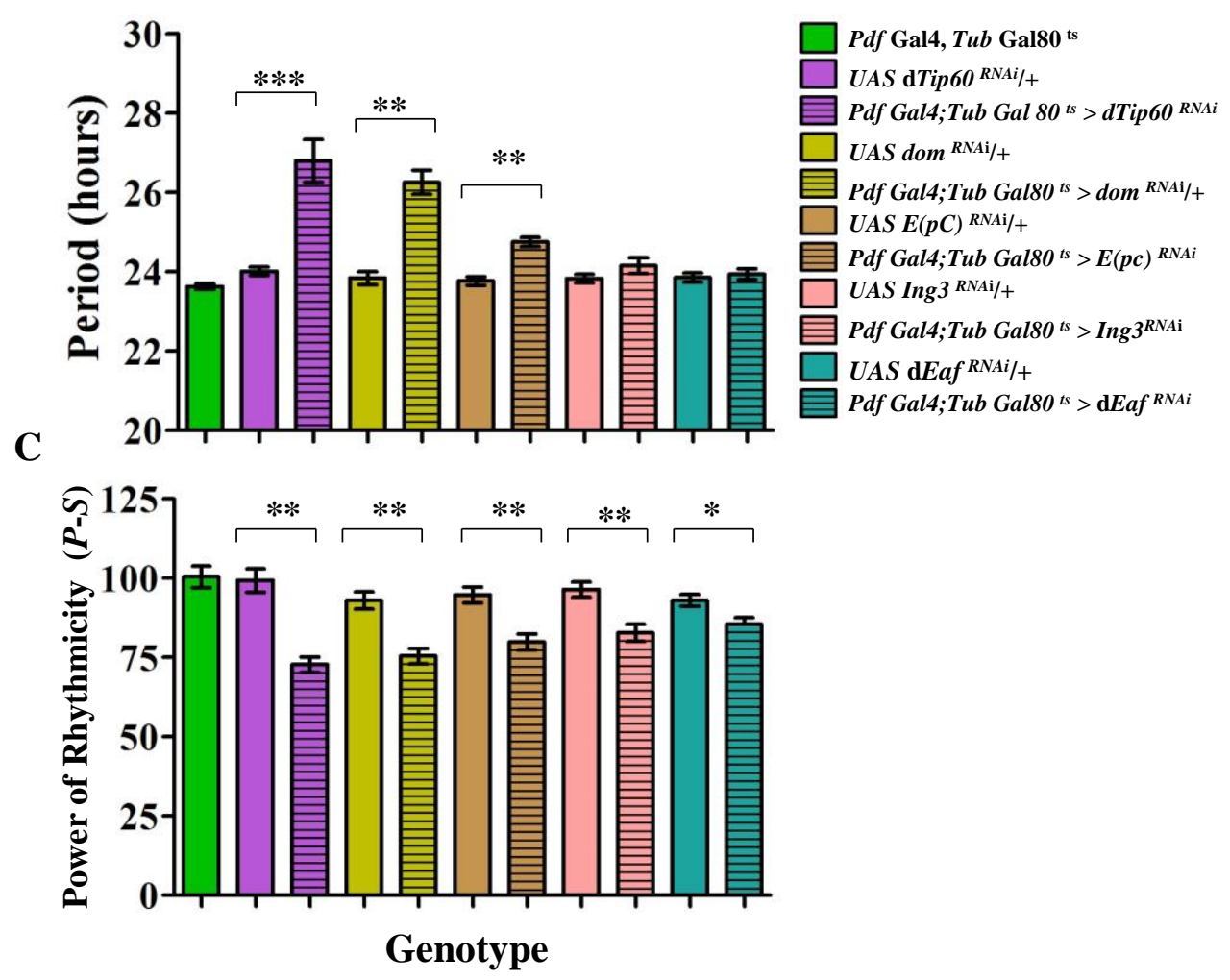

Figure 3.12: Subunits of Nu4Achromatin-remodeling multi protein complex that includes TIP60, contributes to the robust regulation of circadian behavioral rhythms

(A) Schematic of Nu4A chromatin-remodeling complex subunits that were targeted for RNAi knockdown in behavioral assays. (B) Knockdown of individual subunits of TIP60 (Nu4A) complex in lateroventral neurons result in period lengthening. Circadian period determined by $\chi$ square analysis are plotted for each genotype tested. (C) Power of rhythmicity for the tested genotypes. Data in (B) and (C) indicate mean \pm SEM. $* p<0.05$, ** $p<0.01$, *** $p<0.001$ as determined by one-way ANOVA, Tukey post-hoc test. 


\subsection{Tip60 genetically interacts with $C y c$ to regulate circadian rhythms}

It was not surprising to observe that knockdown of individual subunits of Nu4A complex phenocopies knockdown of TIP60 (Table 3-1 and 3-2), since they function together in vivo. While it may not be directly relevant to tease out the circadian functions of each subunit of NU4A complex as there might be compensatory mechanism to set the acetyltransferase activity of the only catalytic subunit TIP60, it is however important to determine the mechanism by which TIP60 would perturb the core molecular clock. In order to gain insights into in vivo functions of dTIP60 in context of circadian clock, a genetic interaction between dTip60 and known clock genes under trans-heterozygous conditions was performed using circadian locomotor behavior assay. Genetic interaction assay in Drosophila is usually utilized to score phenotypes during development or viability as an endpoint. But in this specific case, two mutant alleles were tested simultaneously in a heterozygous state to determine if half -reduction of two of the genes is an important factor in mediating rhythmicity in adult locomotor behavior assay.

To this end, the UAS-dTip $60^{R N A i}$ strain that was previously found to show penetrant phenotypes when targeted in clock neurons was used as bait to fish out other clock genes that will render a stronger phenotype under heterozygous condition in a post emergence behavior assay using percentage rhythmicity as prime criteria. Tip $60^{\mathrm{i}} /+$ denotes the genotype; PdfGal4/ +; UAS-dTip60 $0^{\mathrm{RNAi}} /+$ (strain \#A) in the genetic interaction assays. After standard entrainment in $\mathrm{LD}$ for 5 days at $25^{\circ} \mathrm{C}$, the flies were released into constant dark for seven days to assess the circadian period by $\chi$-square periodogram. The results suggest a strong genetic interaction of dTip60 with a positive activator Cyc and a negative regulator Per (Figure 3.13 A, Table 3-3). The power of rhythmicity was also significantly different for these two clock genes under trans-heterozygous state with dTip60 (Figure $3.13 \mathrm{~B}$ ). One way ANOVA, Dunnet post hoc test revealed an extremely significant interaction (Table 3-4 and 3-5) compared to the parental controls which heterozygous mutants are showing penetrant phenotypes of rhythmicity. $P d f^{01 /}+$ group did not show a strong genetic interaction with Tip $60^{\mathbf{i}} /+$ in the conditions assayed here. Although $\operatorname{Tip}^{\mathrm{i}} \mathrm{i} /+, C l k^{\mathrm{jrk}} /+$ showed a significant difference compared to Tip $60^{\mathrm{i}} /+$, the $\mathrm{Cl} \mathrm{j}^{\mathrm{jrk}} /+$ mutants were already weakly rhythmic and was 
excluded for further analysis. Per in Drosophila is located on X-chromosome and hence, it might be difficult to include gender specific comparison of female flies for locomotor behavior. Since Tip60 is also located in X-chromosome of Drosophila, the observed effect could be masked by random X-chromosome inactivation, which is neither the scope of this assay nor would reveal anything interesting in the context of clock regulation by TIP60. Hence $C y c$ stands out as the strongest candidate from this genetic interaction assay of rhythmic locomotor behavior. It is useful to validate this genetic interaction between $C y c$ and Tip60 further biochemically to get insights into the regulation of circadian clock by TIP60, since CYC is an activator of the core clock machinery and the role of TIP60 in transcriptional activation is well studied (Ikura et al., 2000; Kusch et al., 2004; Jeong et al., 2011).

Table 3-3: Testing genetic interaction using adult locomotor assay at $25^{\circ} \mathrm{C}$ in transheterozygous mutants

\begin{tabular}{|c|c|c|c|c|c|}
\hline Genotype & $n^{1}$ & AR \% ${ }^{2}$ & CR \% ${ }^{3}$ & SR\% ${ }^{4}$ & $\begin{array}{c}\text { Period }(\tau)^{5} \\
\pm \text { SEM (hours) }\end{array}$ \\
\hline$P d f-$ Gal4/ + & 27 & 0 & 11 & 89 & $23.8 \pm 0.6$ \\
\hline$U A S-\mathrm{d} T i p 60^{\mathrm{RNAi}} /+\quad \# \mathrm{~A}$ & 31 & 3 & 5 & 92 & $23.4 \pm 0.5$ \\
\hline $\operatorname{Tip} 60^{\mathbf{i}} /+(\widehat{\jmath})$ & 22 & 10 & 74 & 16 & $26.2 \pm 0.8$ \\
\hline Tip601/+ (+ & 28 & 3 & 72 & 25 & $27.3 \pm 0.2$ \\
\hline $\mathrm{Cyc}^{01} /+$ & 15 & 12 & 68 & 20 & $25.7 \pm 0.1$ \\
\hline Tip60 $1++, C y c^{01} /+$ & 24 & 66 & 26 & 8 & $26.3 \pm 0.6$ \\
\hline$C l k^{\mathrm{jk}} /+$ & 11 & 60 & 28 & 12 & $27.1 \pm 0.9$ \\
\hline Tip60 $/+, C l k^{\mathrm{jrk}} /+$ & 17 & 68 & 18 & 14 & $26.4 \pm 0.2$ \\
\hline $\mathrm{Per}^{01} /+(+)$ & 28 & 21 & 63 & 16 & $24.2 \pm 0.3$ \\
\hline $\operatorname{Tip} 60^{\mathrm{i}} /+$, Per $^{01} /+(+)$ & 16 & 72 & 20 & 8 & $23.7 \pm 1.3$ \\
\hline$P d f^{01} /+$ & 20 & 8 & 16 & 76 & $23.3 \pm 0.4$ \\
\hline $\operatorname{Tip} 60^{\mathbf{i}} /+, P d f^{01} /+$ & 18 & 24 & 34 & 42 & $23.6 \pm 0.7$ \\
\hline
\end{tabular}

${ }^{1} n$ indicates the number of flies tested in each genotype that survived locomotor recordings.

${ }^{2}$ Arrhythmic, ${ }^{3}$ Complex rhythms, ${ }^{4}$ StronglyRhythmic in circadian range.

${ }^{5}$ Period $(\tau)$ in DD is determined by $\chi^{2}$-periodogram analysis.

Table 3-4: Dunnett's multiple comparison of rhythmicity power for Tip60 ${ }^{\mathrm{i}} /+, \mathrm{Cyc}^{01} /+$ transheterozygous combination

\begin{tabular}{|l|l|l|l|}
\hline Genotype & $\begin{array}{l}\text { Difference } \\
\text { of means }\end{array}$ & P value & Significance \\
\hline Tip60 $/+, C y c^{01} /+$ vs Tip60 $/+$ & -47.38 & $<0.0001$ & $* * *$ \\
\hline Tip6O $^{\mathrm{i}} /+, C y c^{01} /+\mathrm{vs} \mathrm{Cyc}^{01} /+$ & -45.50 & $<0.0001$ & $* * *$ \\
\hline
\end{tabular}


Table 3-5: Dunnett's multiple comparison of rhythmicity power for $\mathrm{Tip}^{\mathrm{i}} \mathrm{O}^{\mathrm{i} /+}, \mathrm{Per}^{01} /+$ transheterozygous combination

\begin{tabular}{|c|c|c|c|}
\hline Genotype & $\begin{array}{l}\text { Difference } \\
\text { of means }\end{array}$ & $P$ value & Significance \\
\hline Tip $60^{\mathrm{i} /} /+$, Per $^{01} /+$ vs Tip60 $/+$ & -18.38 & $<0.0001$ & $* * *$ \\
\hline Tip60 $/+$, Per $^{01} /+$ vs $C y c^{01} /+$ & -25.25 & $<0.0001$ & $* * *$ \\
\hline
\end{tabular}

A
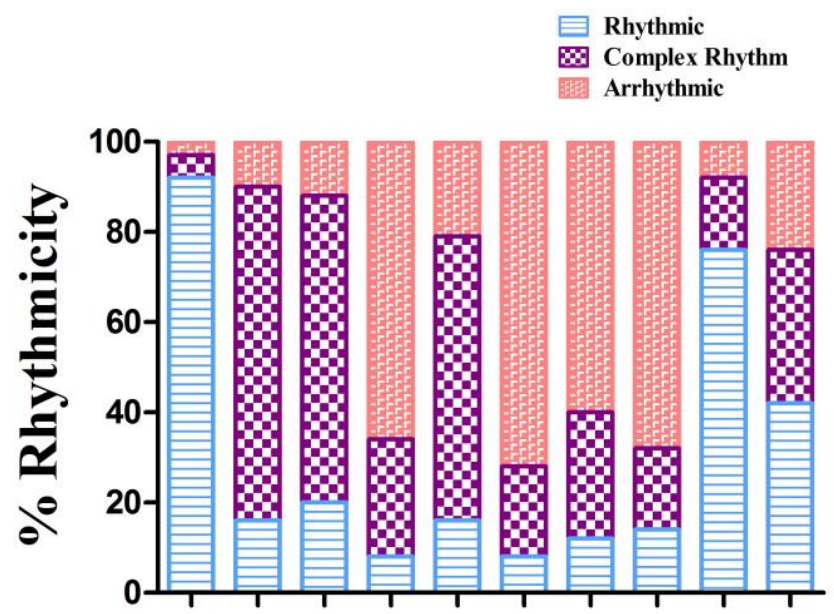

B

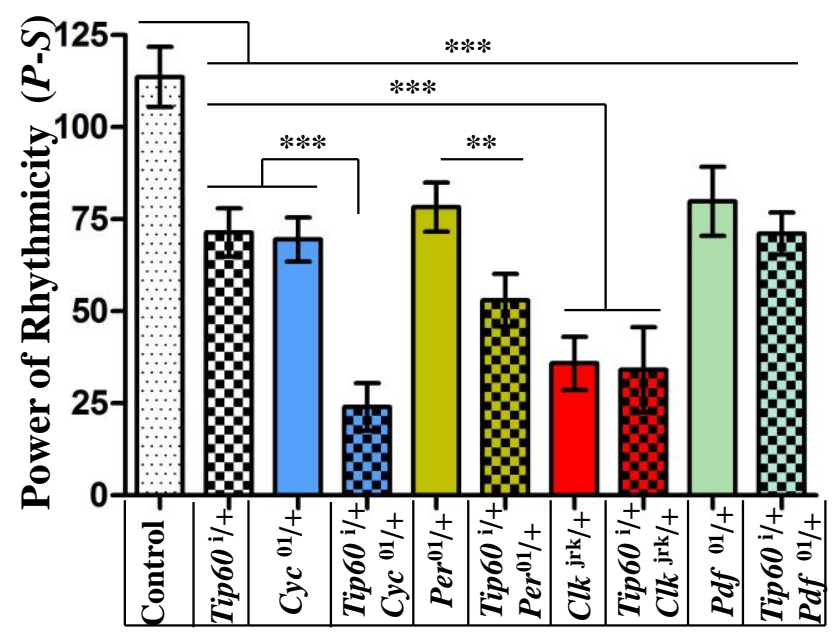

Figure 3.13: Genetic interaction of TIP60 with core clock components

(A) Distribution chart of classified rhythmic percentage in the indicated genotypes under transheterozygous state were determined by $\chi$-square periodogram. (B) Power of rhythmicity as a measure of robustness to determine the strongly interacting genotypes under trans-heterozygous state by locomotor assay. Error bars indicate $95 \%$ confidence interval of mean. $* * p<0.01$, *** $p<0.001$ as determined by one-way ANOVA, Dunnett post-hoc test. 


\subsection{Generation and validation of transgenic strains to express dTIP60-HA tag as a fusion protein}

Although a strong genetic interaction was found between Tip60 and Cyc, it proved technically challenging to study the biochemical basis of TIP60 function in vivo. The antidTIP60 antibody kindly provided by Prof. Ann E. Ehrenhoeffer-Murray (Schirling et al., 2010) was in limited quantity and also the antibody recognizes non-specific epitopes in western blot. The commercially available antibodies have similar problems with recognizing TIP60 specifically at endogenous levels due to recognition of conserved epitopes that are not specific for TIP60 (Urban, 2015). In order to circumvent this problem, a transgenic fly that would express wild type TIP60 with heamagglutinin (HA) tag under the control of UAS was generated. Standard cloning procedures were used to generate constructs of wild type TIP60 that would express HA tag as a fusion protein under UAS (Figure $3.14 \mathrm{~A}$ ). Two independent transgenic insertion fly lines of UAS dTIP60- WT with $\mathrm{N}$ and $\mathrm{C}$ terminal HA tag were generated to serve as a tool for in vivo biochemical assays. These strains were out-crossed for seven generations on a $T M 3, T b$ background and tested for locomotor behavior and expression of Tip60 transcripts. Out of the independent insertion strains, the strain that did not show any abnormalities in circadian behavior upon expression in pacemaker neurons was chosen for further analysis (Figure 3.14 C, left panel). This strain was found to possess a Cterminally tagged HA in TIP60 as a fusion protein (Figure $3.14 \mathrm{~A}$ ). It was not surprising that overexpression of TIP60 does not cause alteration in the clock function, since TIP60 overexpression might not lead to increase in functional complexes with other subunits. However, it serves as a valuable tool to probe the mechanistic underpinning clock regulation by TIP60.

UAS dTIP60-HA was driven using Tubulin promoter and a quantitative real time PCR assay was used to assess the induction of transgene at different temperature regimes. As expected, the responder line driven with Tub-Gal4 showed significantly higher folds of Tip60 transcript levels coinciding with GAL4 activity at higher temperatures compared to the parental controls (Figure 3.14 B). When driven in PDF positive lateroventral neurons, these flies do not show any circadian abnormalities (Figure 3.14 C, left panel). Hence, these flies 
can be utilized as tools to further probe into the circadian function carried on by TIP60. Staining for HA epitope using anti-HA antibody in clock neurons provide clear evidence that the transgenic strain express enough amount of fusion protein (Figure 3.14 C, right panel) that can be used for biochemical assays.

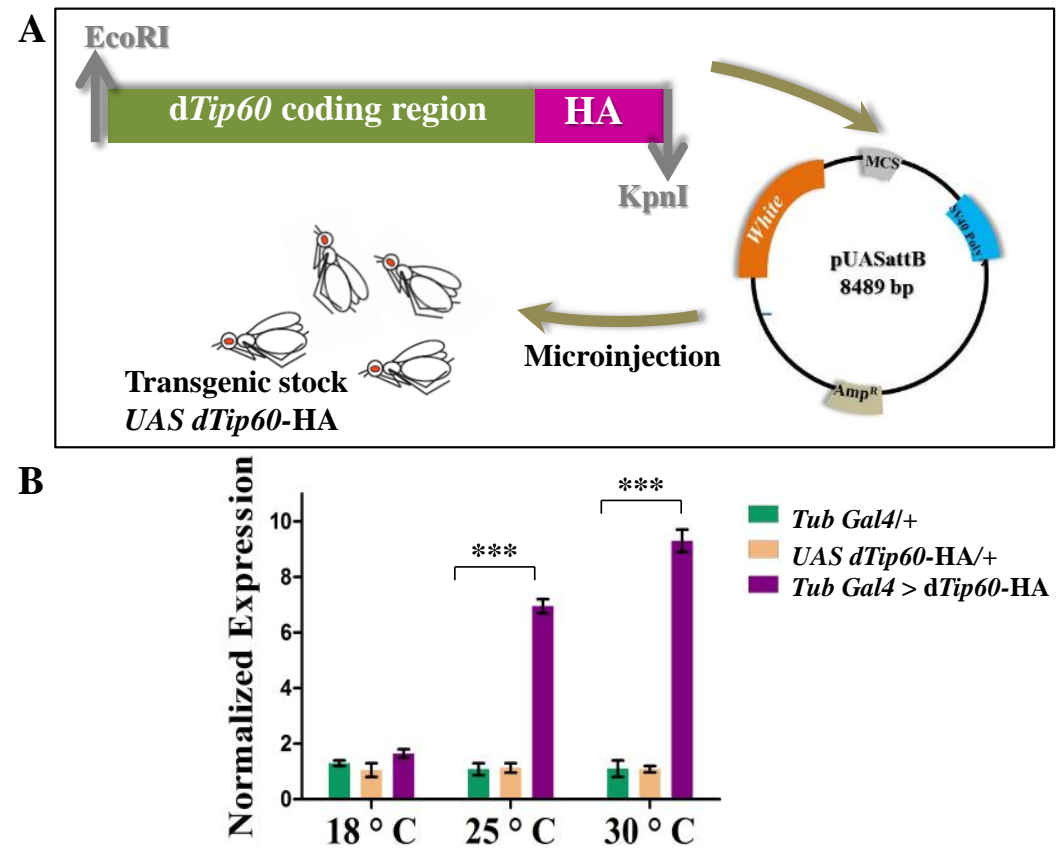

C

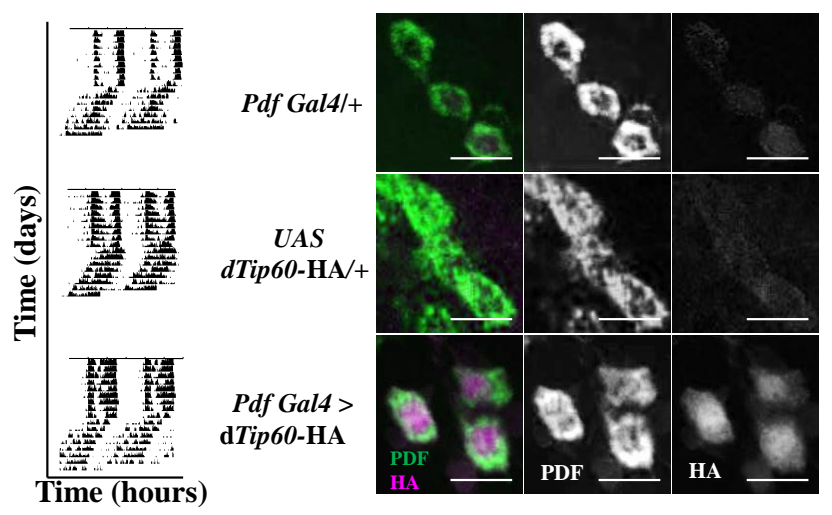

Figure 3.14: Characterization of transgenic strain for the expression of tagged TIP60

(A) Schematic demonstration of constructs used to generate transgenic strain expressing HA-tag fused to TIP60. (B) Quantitative RT-PCR was used to assay the overexpression of transgene by rearing the flies in different temperatures. $* * * p<0.001$ was determined by one-way ANOVA, Dunnet post-hoc test. (C) Left panel shows representative actograms of flies expressing TIP60- 
HA in lateroventral clock neurons using Pdf-Gal4 driver and their controls. Right panel shows representative confocal images of indicated genotype stained with anti-HA and anti-PDF antibody. Scale bars represent $20 \mu \mathrm{m}$.

\subsection{TIP60 localizes near CLK/CYC binding sites on clock controlled genes}

Since knockdown of Tip60 or misexpression of catalytic mutant version TIP60 E431Q affects the circadian oscillator function and also TIP60 strongly interacts genetically with CYC in a locomotor assay, it is clear that TIP60 may interact with the CLK/CYC activator complex at its target genes to regulate rhythmic expression. To examine if TIP60 binds to regulatory regions of CLK/CYC in the target genes, TIP60 binding to the promoters of Per and Tim was assayed using Chromatin immuno-precipitation (ChIP). UAS dTIP60-HA responder was directed ubiquitously using Tub-Gal4 allowing the investigation of TIP60 binding to CLK/CYC target genes.

ChIP followed by quantitative PCR analysis of flies expressing TIP60-HA revealed that over circadian cycle, TIP60 is bound to the E-box regions at Per and Tim promoter (Figure $3.15 \mathrm{~B})$. Control ChIP experiments were performed on the UAS parental flies that do not express HA tag. The relative locations of primers used for testing E-box binding are shown in Figure 3.15 A, the fifth E-box region chosen in Per promoter is located within a 69 bp region critical for generating rhythmic expression of $\mathrm{Per}$ known as clock regulatory sequence (CRS) (Menet et al., 2010). The E-box on Tim locus has been previously identified as a CLK binding site (Hao et al., 1997). The peak of TIP60 binding to promoter regions of Per and Tim appears to be at CT16 from the results. However, acquiring chromatin fragments of desired length and quality for a ChIP assay proved to be was technically limiting due to the presence of hard cuticle in adult flies. This also compromises the resolution of time axis in this assay with use of large group of flies for each time point. Hence the real peak of TIP60 binding to E-boxes of clock controlled genes might be somewhere between CT 10 and CT 16 which corresponds to the maximal expression of key transcripts like Per and Tim (refering control dataset in Figure 3.9 and Tim data set in Figure 3.11). The non specific binding was controlled for by assaying an intergenic region in the 3' region and performing appropriate 
normalization. This result establishes the hypothesis that TIP60 has an activating function in concert with CLK/CYC activator complex to regulate circadian clock.
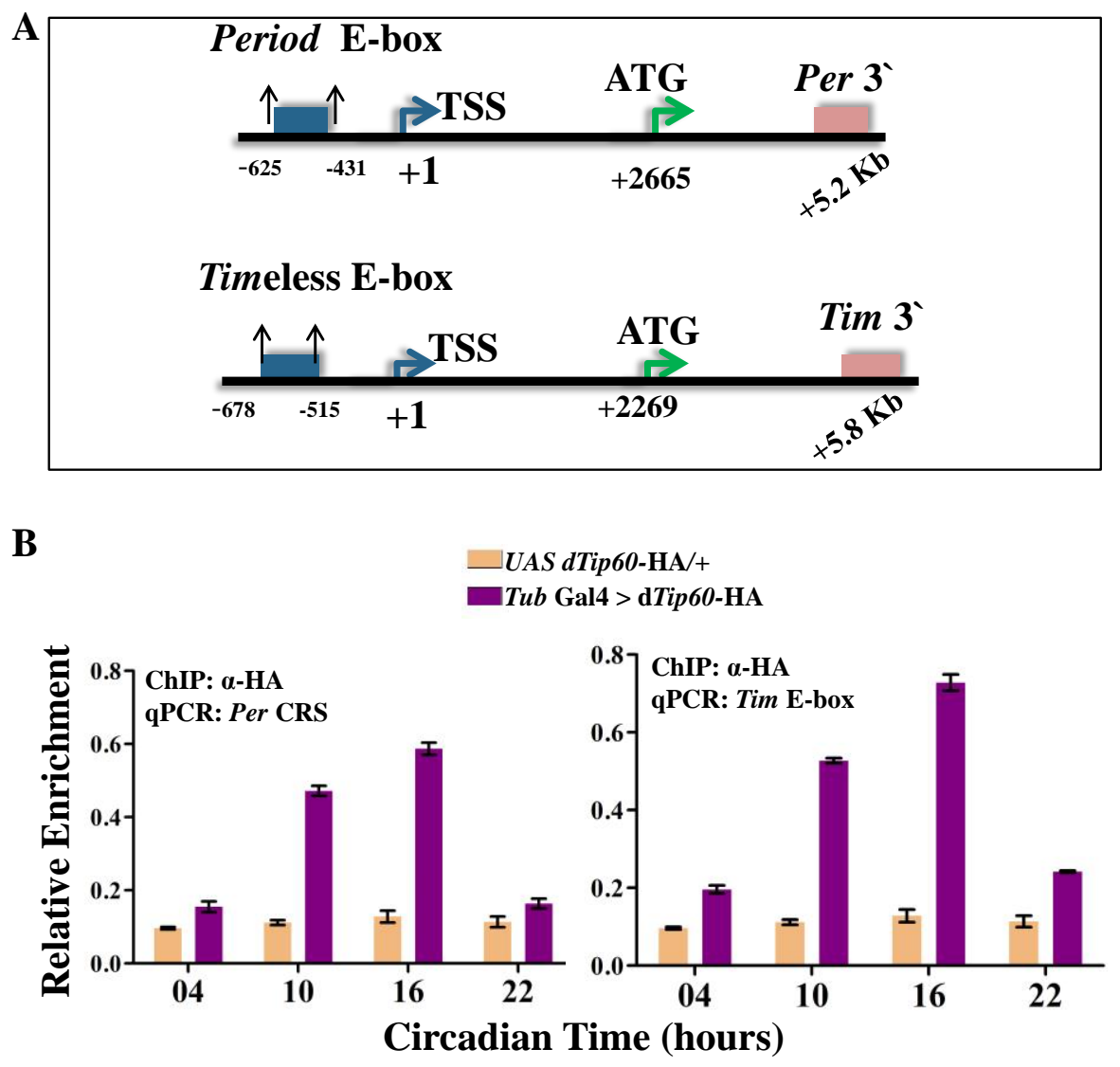

Figure 3.15: TIP60 localizes to CLK/CYC binding sites on Per and Tim genes with spatial and temporal proximity

(A) Schematic of primer locations used for Chromatin-IP analysis on Per and Tim loci. Regions are numbered relative to the transcription start site (TSS). (B) Chromatin immunoprecipitation assay with transgenic flies which overexpress HA fused to TIP60 under the control of Tubulin promoter. Anti-HA antibody was used to pull down HA-tagged TIP60 at indicated time points. Non-specific binding was detected by amplifying an intergenic region in the 3 ' end of the corresponding gene and used appropriately to subtract signal from Per clock regulatory sequence (CRS) containing E-box elements and Tim E-box elements. Error bars represent standard error of mean for four independent biological replicates. 
A

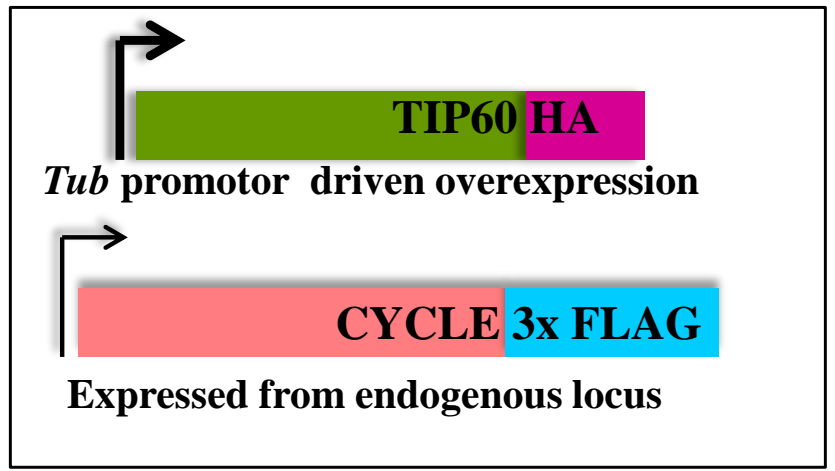

B

\begin{tabular}{|l|l|l|l|l|}
\cline { 2 - 5 } \multicolumn{1}{c|}{} & \multicolumn{2}{|c|}{ Input } & \multicolumn{2}{c|}{\begin{tabular}{c} 
IP: \\
\multicolumn{1}{c|}{-FLAG }
\end{tabular}} \\
\hline Tub Gal4>dTip60HA & + & & + & \\
\hline $\begin{array}{l}\text { Tub Gal4>dTip60HA/ } \\
\text { Cyc-3x FLAG }\end{array}$ & & + & & + \\
\hline
\end{tabular}

\begin{tabular}{|l|l|l|l|l|}
\cline { 2 - 5 } \multicolumn{1}{c|}{} & \multicolumn{2}{c|}{ Input } & \multicolumn{2}{c|}{$\begin{array}{c}\text { IP: } \\
\text { a-HA }\end{array}$} \\
\hline Cyc-3x FLAG & + & & + & \\
\hline $\begin{array}{l}\text { Tub Gal4>dTip60HA/ } \\
\text { Cyc-3x FLAG }\end{array}$ & & + & & + \\
\hline
\end{tabular}

a-FLAG
(CYC)

a-HA

(TIP60)
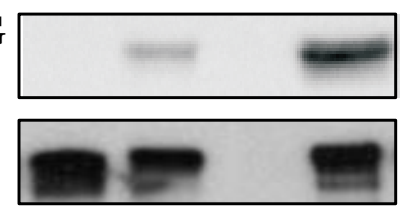

$\alpha-$ TUB
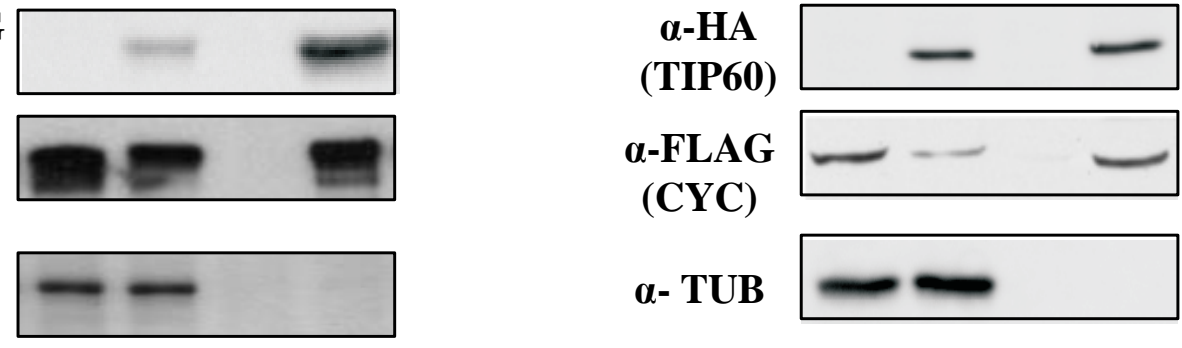

\section{Figure 3.16: In vivo interaction of CYC and TIP60}

(A) Schematic of transgenic constructs present in the flies used for in vivo immunoprecipitation. Overexpression of TIP60-HA was directed ubiquitously using a Tubulin driver. 3x FLAG tag was expressed from endogenous locus. (B) Western blots showing results of reciprocal coimmunoprecipitation to detect in vivo interaction of TIP60 with CYC in whole heads at CT 12. Protein lysate from heads were immunoprecipitated with anti-HA antibody to pull down TIP60 and anti-FLAG antibody to pull down CYC. The responder strain which does not produce any fusion protein was treated as negative control. Data shown are representative blots of three biological replicates. 


\subsection{TIP60 interacts with CYC in vivo}

Since a strong genetic interaction of TIP60 and CYC prompted to further explore the mechanistic regulation of the clock by TIP60, it would be essential to test if TIP60 interacts with CYC biochemically. Transgenic flies that express TIP60- HA under Tub-Gal4 promoter was crossed to Cyc-3xFLAG strain which expressed FLAG tagged CYC under the control of its endogenous promoter. Such a fly genotype would enable a reciprocal coimmunoprecipitation to test the interaction between TIP60 and CYC. The constructs present in experimental flies are shown in Figure 3.16 A.

The flies were entrained for 4 days in $\mathrm{LD}$ at $25^{\circ} \mathrm{C}$ and collected at $\mathrm{CT} 12$ on the second day of DD. Protein lysates were prepared from fly heads and immunoprecipitated using antiHA antibody to pull down TIP60-HA and anti-FLAG antibody to pull down CYC-3x FLAG. Immunoprecipitated complexes were subjected to western blotting to detect interactions between TIP60 and CYC. Flies that do not express both HA and FLAG tag epitopes were used as controls (Figure $3.16 \mathrm{~B}$ ). Western blots of reciprocal co-IPs indicated a strong interaction between TIP60 and CYC at CT 12 (Figure 3.16 B). Taken together, these results demonstrate a further validation of the genetic interaction scored between TIP60 and CYC, revealing new insights about TIP60 function in the circadian clock. This interaction is perhaps the reason for observing a strong circadian dysfunction through behavioral assays and gene expression analysis. 


\section{Discussion}

\subsection{Ubiquitous presence of TIP60 and a role in circadian clock}

The presence of circadian clocks in simple organisms such as cyanobacteria (Johnson et al., 1996) provides a hint that such clocks may arose very early in the history of life (DeCoursey, 2004). The pervasive evolution of circadian clocks might be due to the adaptive advantage it confers to the organism that harbors such a functional clock. The widely accepted Pittendrigh's "escape from light hypothesis" speculate that primitive organisms must have evolved circadian clocks in order to avoid the harmful effects of UV induced DNA damage during the day time. This adaptive pressure should have thence forced critical biological processes such as reproduction (replication and mitotic division of cells) to be confined towards night. Interestingly, the negative regulator of the mammalian circadian clockwork is represented by Cryptochrome genes that have likely evolved from light-induced DNA repair enzymes (DNA photolyases) (Rosato et al., 2001; Gaddameedhi et al., 2012). Moreover, TIP60 has a clear role in DNA damage response for which the acetyltransferase activity is important (Kranz et al., 2008; $\mathrm{Xu}$ et al., 2011). In a similar context TIP60 must have evolved to regulate the circadian clock as an adaptive response to DNA damage.

TIP60 being an acetyltransferase is a necessary enzyme present in adult tissues at low expressed levels, maintaining a regulatory cellular function in combination with different target proteins. dTIP60 is present in adult Drosophila brain in an ubiquitous manner (Lorbeck et al., 2011; Sarthi and Elefant, 2011). Also results from staining experiments revealed a ubiquitous presence of TIP60 in the adult Drosophila brain (Figure 3.3). TIP60 was already known to be expressed in ventro lateral neurons in Drosophila (Pirooznia et al., 2012). Previous work from the Eichele's laboratory reveals presence of TIP60 in mouse SCN and also in adult hippocampus (Urban, 2015). Hence it is clear that TIP60 might act differently with target proteins to exert several processes depending on the cellular context. Ubiquitous presence of TIP60 also hints at the possibility that it might be playing important role in cellular mechanisms including the circadian clock. Hence, the potent role of TIP60 as a co-activator of circadian activators is strengthened. 


\subsection{Establishing TIP60 in the circadian molecular loop}

Data demonstrated in this thesis clearly shows that TIP60 regulates the circadian clock both behaviorally and molecularly (Figure 3.5 and 3.13). There is a clear reduction in the oscillating levels of core clock genes (Figures 3.7 and 3.8). TIP60 acetylates the $\mathcal{E}$-amino group of substrate lysine residues with the help of cofactor coenzyme-A, which then provides the acetyl moiety. The catalytic mutant overexpression $\left(T I P 60^{E 431 Q}\right)$ in clock neurons produces a drastic phenotype probably due to the inability to deprotonate the $\mathcal{E}$-amino group of lysine substrates (Decker et al., 2008). This renders the overexpressed protein to act in a dominant negative fashion and thereby bring a phenotype towards loss of TIP60 function (Lorbeck et al., 2011). However TIP60 itself is not expressed under the control of circadian clock. This provides an interesting situation to place TIP60 in the activation loop of the circadian clock, since a genetic and biochemical interaction was obviously demonstrated (Figures 3.11 and 3.14). The use of transgenic flies to assay binding of TIP60 to the promoter regions of Per and Tim unequivocally indicates that TIP60 participates with the circadian activators to turn on gene expression of core clock controlled genes as demonstrated in Figure 4.1 A.

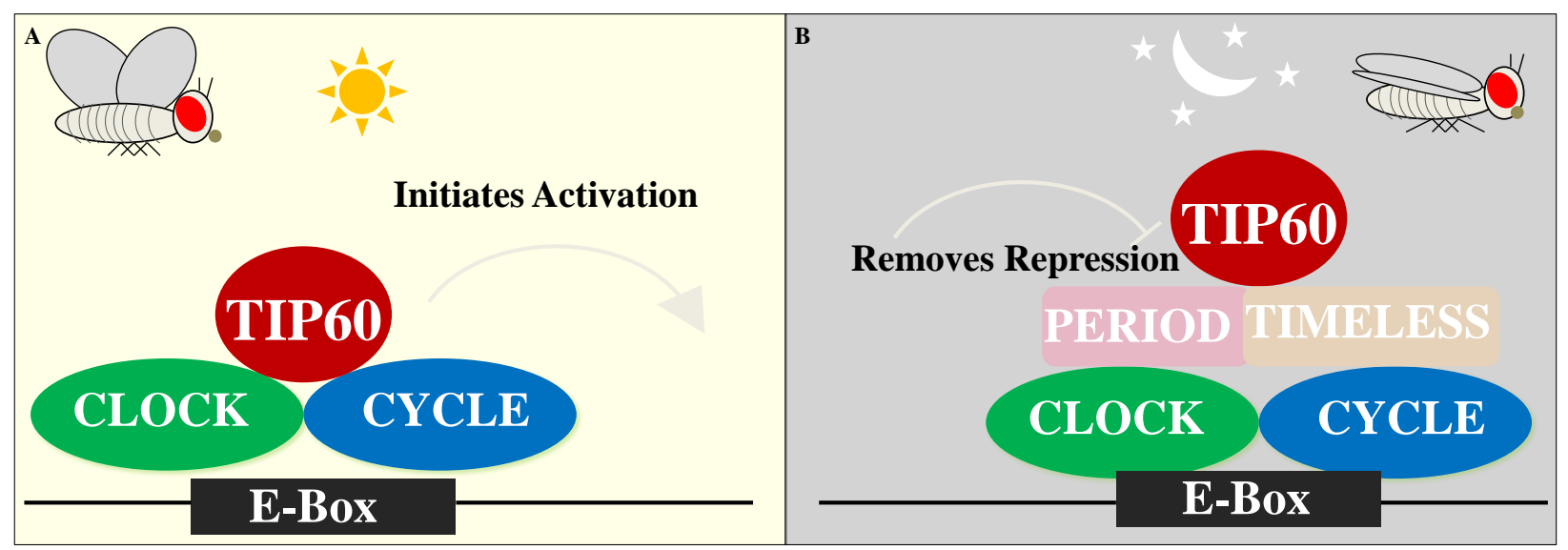

Figure 4.1: A model of TIP60 induced activation of circadian clock and balance by repressors

This model was devised by placing TIP60 on the existing TTFL paradigm. (A) The recruitment of CLOCK-CYCLE heterodimer on the E-boxes could be initiated by TIP60 during the day in order to turn on circadian gene transcription. (B) The negative repressors such as PERIOD and TIMELESS gain sufficient stability to inhibit the activation step during the night. TIP60 could also be a part of reactivation mechanism thereby sending signals to remove the repressors. 
It is also not very clear from the existing TTFL model, how the repressors are removed effectively from the E-boxes. The auxiliary loop does not completely qualify to fine tune the removal of repressor complex from chromatin, in anticipation of the next circadian cycle. Although there may be several deacetylases involved in regulating this step, TIP60 qualifies as a unique candidate to induce activation by removing repressor complex as shown in Figure 4.1 B. This process could be indirectly signaled by the competition between several deacetylases and TIP60, which remains to be scrutinized in detail.

The main goal of performing a genetic interaction assay is to probe the potential substrate of TIP60 in context of circadian clock. Acetyltransferase TIP60 being important for several cellular functions, it is not surprising that any other genetic screen had failed to identify its role in the circadian clock. Since a strong genetic interaction between CYC and TIP60 was further validated with biochemical assay, the potent substrate is not very far from reach. Investigating the mammalian clock system reveals, the homolog of CYC, BMAL1 is acetylated. However, acetylation of BMAL1 occurs in the lysine residue present in transactivation domain and is mediated by acetyltransferase activity of the circadian activator CLOCK (Doi et al., 2006). Interestingly, CYC lacks the C-terminal transactivation domain although other regions are well conserved (Figure 4.2). This raises questions on how CYC in Drosophila is capable of activating circadian transcription. Earlier work from Allada's laboratory have shown that ectopic CLOCK overexpression can induce rhythmic gene expression (Zhao et al., 2003). However it is also known that this effect is mediated only by binding with heterodimeric partner of CLK, i.e., CYC (Taylor and Hardin, 2008).

Expression of CYC during development was shown to be necessary for functioning of a ticking clock in the adult in another study (Goda et al., 2011). The effector, PER appears not to be crucial during development to harbor a functioning adult locomotor rhythm. Hence adult clock function relies on CLK/CYC during development than on the oscillatory repressors. It should however be noted that the activators themselves are not expressed with robust rhythmicity as the repressors either during development or in the adult stages (Houl et al., 2006). Hence an enzyme expressed in non-rhythmic fashion could switch roles to generate rhythmic patterns of gene expression by modifying one or several of its substrates. 


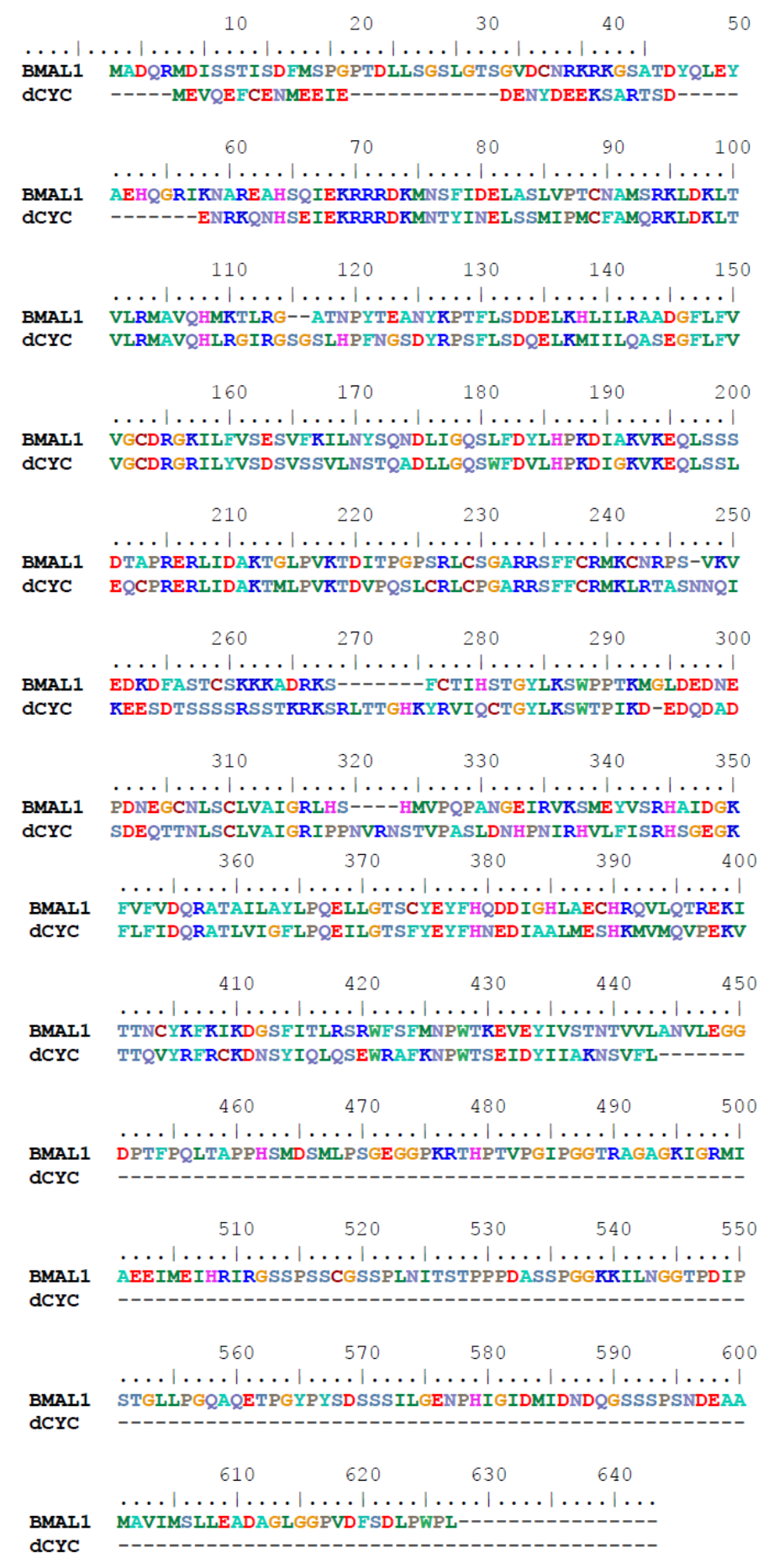

Figure 4.2: Pair-wise sequence alignment of mouse BMAL1 and Drosophila CYCLE

Full length pair-wise sequence alignment of BMAL1 and its homolog CYCLE reveals that transactivation domain towards the carboxyl terminal is absent in Drosophila. CYC protein of the order Diptera is shorter than its mammalian homolog. 


\subsection{Lysine acetylation in CYC as a potent regulator of circadian transcription}

Now the focus for future perspective lies clearly in finding acetylation patterns in the putative substrate CYC. Comparison of crystal structure of BMAL1 (Huang et al., 2012) shows strongly conserved lysine residues exposed on the surface in the basic helix-loop-helix region (personal observation). Highly conserved lysine residues can also be skimmed in Figure 4.2. Hence, investigating if any of these lysine residues would be acetylated in $\mathrm{CYC}$ for the regulation of circadian clock would be a feasible and achievable goal. However, it is also mandatory to detect rhythmic patterns of acetylation in the substrate CYC and correlate that to the rhythmic occupancy in the E-box regions of clock controlled genes. Future work will reveal novel insights into the regulation of circadian transcriptional activation by post translational modification of a circadian activator heterodimer. The abundance of CYC does not vary during the day (Rutila et al., 1998), suggesting that post translational modification by acetylation is an effective way to act as a sensor to start transcription or recruitment of other molecular machinery needed to initiate transcription.

It should also be taken into account that CYC always functions as a heterodimer with its binding partner CLK in a 1:1 stoichiometry (Lee et al., 1999). This raises the possibility that interaction of TIP60 and CYC might enable rapid recruitment of CLK-CYC heterodimer on the chromatin. Alternatively, TIP60 could potentially acetylate CLK or enable the recruitment of other acetyltransferases and chromatin remodeling enzymes in a time dependent fashion at particular clock-controlled loci harboring E-boxes. CLK in Drosophila is known to be the potent activator of circadian transcription and hence it will be interesting to investigate if acetylation of CYC will result in potential modification of CLK at structural level in the macromolecular complex bound to DNA. There were no studies to date, demonstrating the acetyltransferase function of CLK in Drosophila and hence investigating if TIP60 is a potent acetyltransferase in the Drosophila circadian clock will shed more insights into the molecular mechanisms underlying the generation of circadian period. This works sets a clear ground for investigating the potent mechanistic role of TIP60 in fine tuning of circadian clock by acetylation. 


\subsection{Functions of TIP60 converging into the circadian clock}

As expected, TIP60 possess widely known non-circadian functions being an acetyltransferase required for cell growth and metabolism. The best known functions of TIP60 are in apoptotic regulation with HIV Tat (Col et al., 2005) and DNA damage response (Ikura et al., 2000; Kusch et al., 2004). In Drosophila, TIP60 regulates development of nervous system (Lorbeck et al., 2011), axonal transport (Johnson et al., 2013) cognitive functions including memory (Johnson et al., 2013; Xu et al., 2014). All of these non-circadian functions could be directly or indirectly related to the functioning of a robust circadian clock in the organism.

In order to understand the functional relevance of TIP60 multiprotein complex in the circadian clock, knockdown of individual subunits of Nu4A complex in the clock neurons was performed. These results strongly indicate that TIP60 might functions as a multi protein complex even in the circadian context (Figure 3.12). Results of the longevity assay in flies with knockdown of Tip60 in pacemaker clock neurons indicate that TIP60 plays an important role in maintaining general health of the organism with a robust circadian clock. A recent study reported the involvement of class II histone deacetylases in the negative loop of circadian clock (Fogg et al., 2014). Investigation of the requirement of deacetylases in Drosophila circadian clock might fill the gap in current understanding of the TTFL model. In particular, it will be of great importance to know which class of deacetylases function antagonistic to TIP60, such that devising strategies to intervene in certain disease states would be possible. In addition, it will be ideal and convenient to screen other members of the MYST family acetyltransferases in circadian assays using Drosophila.

Recent reports indicate the manifestation of circadian dysfunction in several cognitive and psychiatric diseases (Zordan and Sandrelli, 2015). Taken together, the data from this thesis and of multiple independent studies performed across different species it becomes very clear that circadian dysfunction can be both a cause and consequence of devastating diseases including cancer. The involvement of TIP60 in the circadian clock would thus enable the understanding of mechanisms that converge into or diverge from the core molecular clock. 


\subsection{Integrating TIP60 within the network model of circadian clock}

The central theme of this thesis work is focused on the fact that circadian behavioral rhythms are abolished by the knockdown of Tip60 or overexpression of catalytic mutant version

of TIP60 ${ }^{E 431 Q}$ in central pacemaker neurons under the control of Pdf promoter (Table 3-1 and Table 3-2). However, the Drosophila clock neuron network is organized into multiple independent neuronal oscillators (Yao and Shafer, 2014) which results in a network giving rise to emergent rhythmic activity (Dissel et al., 2014) that interact both at the cellular and molecular level (Weiss et al., 2014). Since Tip60 is expressed at low levels throughout the adult Drosophila head (Contrino et al., 2012) and also expressed in nervous system (Lorbeck et al., 2011; Xu et al., 2014) it is highly likely that TIP60 will act in a cell specific manner in different network of oscillators. It will be interesting to evaluate the properties of independent oscillator network by manipulating TIP60 in different neurons as shown in Figure 4.3.

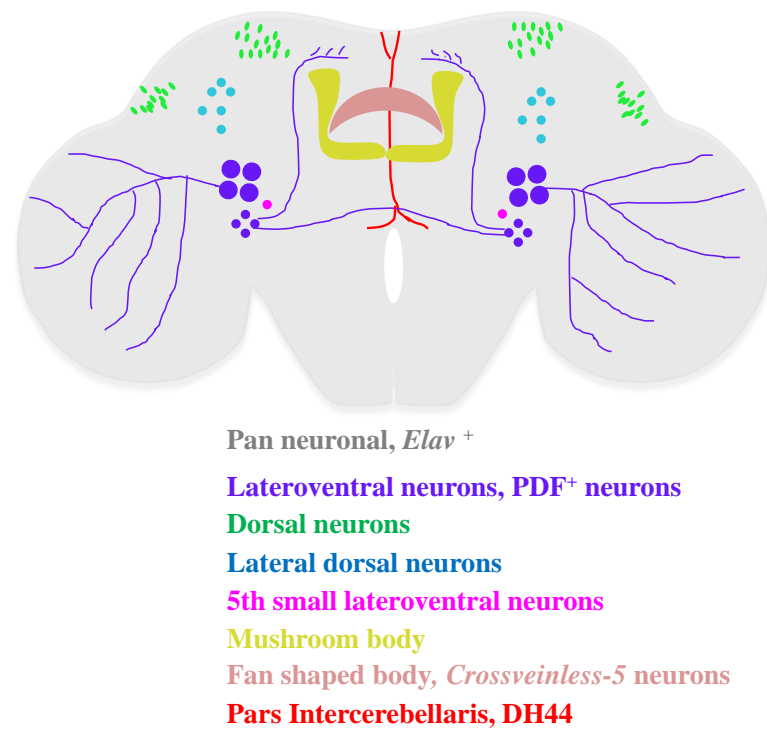

Figure 4.3: Proposed manipulations of circadian network in Drosophila brain

Schematic representation of Drosophila brain demonstrating well characterized independent circadian oscillator networks. Lateroventral neurons, dorsolateral neurons, dorsal neurons and the $5^{\text {th }}$ small lateroventral neurons are part of the independent autonomous circadian network. Mushroom body, fan shaped body (Dissel et al., 2015) and pars intercerebellaris (Cavanaugh et al., 2014) are parts of the output circuit that mediate circadian locomotor activity and sleep. Manipulating Tip60 in these regions will shed light on the response of network integration mediated by TIP60. 
So far, the clock network and output circuits those were described relates to one primary entity of input or zeitgeber, namely light. Circadian clocks are temperature and nutrition compensated while they entrain to cues such as food (Mistlberger and Antle, 2011) and external temperature (Bodenstein et al., 2012). Given that TIP60 is well conserved among eukaryotes and is a potent acetyltransferase that can activate circadian transcription, it will be a worthy effort to explore the involvement of TIP60 in temperature entrainment and temperature compensation. TIP60 being an enzyme, it functions effectively and acetylates its substrates at ambient temperature. In this context, it will be valuable to utilize the power of computer simulations and mathematical modeling to predict how TIP60 can generate circadian period in the existing TTFL model and compensate temperature in a circadian fashion.

Recent evidence on the presence of non-TTFL clocks such as oxidation-reduction rhythms by peroxiredoxins (Edgar et al., 2012) have reiterated the importance of circadian clocks as a pervasive way to adapt better from harmful situation present in the environment. Peroxiredoxin rhythms are conserved in Drosophila and mammals, however they oscillate in a different phase to the that of the TTFL clock indicating co-evolution of a primitive clock (Edgar et al., 2012). Circadian pacemakers operate by the balance of positive and negative feedback loop. The interplay of positive activators and negative repressors bring a gamut of metabolic and physiological changes that enable organisms to adapt well in their niche. The evolutionary significance of acetylation as a means of gene transcription in eukaryotes confirms that such mechanisms are not only conserved but are very essential for the survival of organisms. Hence the role of a well conserved acetyltransferase such as TIP60 can be well understood in the context of circadian clock and general survival of Drosophila and other eukaryotes.

The work presented in this thesis lays strong foundation for the further investigation of molecular mechanisms that orchestrate the circadian clock in a robust yet adaptive and plastic manner, with a major emphasis on TIP60 as a central player of the circuit. 


\section{References}

Abruzzi KC, Rodriguez J, Menet JS, Desrochers J, Zadina A, Luo W, Tkachev S, Rosbash M (2011) Drosophila CLOCK target gene characterization: implications for circadian tissuespecific gene expression. Genes Dev 25:2374-2386.

Aschoff J (1963) Comparative physiology: diurnal rhythms. Annu Rev Physiol 25:581-600.

Bell-Pedersen D, Cassone VM, Earnest DJ, Golden SS, Hardin PE, Thomas TL, Zoran MJ (2005) Circadian rhythms from multiple oscillators: lessons from diverse organisms. Nat Rev Genet 6:544-556.

Bodenstein C, Heiland I, Schuster S (2012) Temperature compensation and entrainment in circadian rhythms. Phys Biol 9:036011.

Brand AH, Perrimon N (1993) Targeted gene expression as a means of altering cell fates and generating dominant phenotypes. Development 118:401-415.

Cavanaugh DJ, Geratowski JD, Wooltorton JRA, Spaethling JM, Hector CE, Zheng X, Johnson EC, Eberwine JH, Sehgal A (2014) Identification of a circadian output circuit for rest:activity rhythms in Drosophila. Cell 157:689-701.

Col E, Caron C, Chable-Bessia C, Legube G, Gazzeri S, Komatsu Y, Yoshida M, Benkirane M, Trouche D, Khochbin S (2005) HIV-1 Tat targets Tip60 to impair the apoptotic cell response to genotoxic stresses. EMBO J 24:2634-2645.

Contrino S et al. (2012) modMine: flexible access to modENCODE data. Nucleic Acids Res 40:D1082-8.

Creaven M, Hans F, Mutskov V, Col E, Caron C, Dimitrov S, Khochbin S (1999) Control of the histone-acetyltransferase activity of Tip60 by the HIV-1 transactivator protein, Tat. Biochemistry 38:8826-8830.

Cyran SA, Buchsbaum AM, Reddy KL, Lin M-C, Glossop NRJ, Hardin PE, Young MW, Storti RV, Blau J (2003) vrille, Pdp1, and dClock form a second feedback loop in the Drosophila circadian clock. Cell 112:329-341.

Decker PV, Yu DY, Iizuka M, Qiu Q, Smith MM (2008) Catalytic-site mutations in the MYST family histone Acetyltransferase Esa1. Genetics 178:1209-1220.

DeCoursey PJ (2004) The Behavioral Ecology and Evolution of Biological Timing Systems. In: Chronobiology: Biological Timekeeping (Dunlap JC, Loros JJ, DeCoursey PJ, eds), pp 27-66. Sunderland, Mass: Sinauer Associates. 
Dissel S, Angadi V, Kirszenblat L, Suzuki Y, Donlea J, Klose M, Koch Z, English D, WinskySommerer R, van Swinderen B, Shaw PJ (2015) Sleep restores behavioral plasticity to Drosophila mutants. Curr Biol 25:1270-1281.

Dissel S, Hansen CN, Özkaya Ö, Hemsley M, Kyriacou CP, Rosato E (2014) The logic of circadian organization in Drosophila. Curr Biol 24:2257-2266.

Doi M, Hirayama J, Sassone-Corsi P (2006) Circadian regulator CLOCK is a histone acetyltransferase. Cell 125:497-508.

Edgar RS, Green EW, Zhao Y, van Ooijen G, Olmedo M, Qin X, Xu Y, Pan M, Valekunja UK, Feeney KA, Maywood ES, Hastings MH, Baliga NS, Merrow M, Millar AJ, Johnson CH, Kyriacou CP, O’Neill JS, Reddy AB (2012) Peroxiredoxins are conserved markers of circadian rhythms. Nature 485:459-464.

Emery P (2007) RNA extraction from Drosophila heads. Methods Mol Biol 362:305-307.

Fogg PCM, O’Neill JS, Dobrzycki T, Calvert S, Lord EC, McIntosh RLL, Elliott CJH, Sweeney ST, Hastings MH, Chawla S (2014) Class IIa histone deacetylases are conserved regulators of circadian function. J Biol Chem 289:34341-34348.

Gaddameedhi S, Reardon JT, Ye R, Ozturk N, Sancar A (2012) Effect of circadian clock mutations on DNA damage response in mammalian cells. Cell Cycle 11:3481-3491.

Goda T, Mirowska K, Currie J, Kim M-H, Rao NV, Bonilla G, Wijnen H (2011) Adult circadian behavior in Drosophila requires developmental expression of cycle, but not period. PLoS Genet 7:e1002167.

Granados-Fuentes D, Prolo LM, Abraham U, Herzog ED (2004) The suprachiasmatic nucleus entrains, but does not sustain, circadian rhythmicity in the olfactory bulb. J Neurosci 24:615-619.

Hao H, Allen DL, Hardin PE (1997) A circadian enhancer mediates PER-dependent mRNA cycling in Drosophila melanogaster. Mol Cell Biol 17:3687-3693.

Hermann C, Saccon R, Senthilan PR, Domnik L, Dircksen H, Yoshii T, Helfrich-Förster C (2013) The circadian clock network in the brain of different Drosophila species. J Comp Neurol 521:367-388.

Houl JH, Ng F, Taylor P, Hardin PE (2008) CLOCK expression identifies developing circadian oscillator neurons in the brains of Drosophila embryos. BMC Neurosci 9:119.

Houl JH, Yu W, Dudek SM, Hardin PE (2006) Drosophila CLOCK is constitutively expressed in circadian oscillator and non-oscillator cells. J Biol Rhythms 21:93-103. 
Huang N, Chelliah Y, Shan Y, Taylor CA, Yoo S-H, Partch C, Green CB, Zhang H, Takahashi JS (2012) Crystal structure of the heterodimeric CLOCK:BMAL1 transcriptional activator complex. Science 337:189-194.

Husse J, Leliavski A, Tsang AH, Oster H, Eichele G (2014) The light-dark cycle controls peripheral rhythmicity in mice with a genetically ablated suprachiasmatic nucleus clock. FASEB J 28:4950-4960.

Ikura T, Ogryzko VV, Grigoriev M, Groisman R, Wang J, Horikoshi M, Scully R, Qin J, Nakatani Y (2000) Involvement of the TIP60 histone acetylase complex in DNA repair and apoptosis. Cell 102:463-473.

Im SH, Taghert PH (2010) PDF receptor expression reveals direct interactions between circadian oscillators in Drosophila. J Comp Neurol 518:1925-1945.

Ivanchenko M, Stanewsky R, Giebultowicz JM (2001) Circadian photoreception in Drosophila: functions of cryptochrome in peripheral and central clocks. J Biol Rhythms 16:205-215.

Jeong KW, Kim K, Situ AJ, Ulmer TS, An W, Stallcup MR (2011) Recognition of enhancer element-specific histone methylation by TIP60 in transcriptional activation. Nat Struct Mol Biol 18:1358-1365.

Johnson AA, Sarthi J, Pirooznia SK, Reube W, Elefant F (2013) Increasing Tip60 HAT levels rescues axonal transport defects and associated behavioral phenotypes in a Drosophila Alzheimer's disease model. J Neurosci 33:7535-7547.

Johnson CH (2004) Precise circadian clocks in prokaryotic cyanobacteria. Curr Issues Mol Biol 6:103-110.

Kadener S, Menet JS, Schoer R, Rosbash M (2008) Circadian transcription contributes to core period determination in Drosophila. PLoS Biol 6:e119.

Kamine J, Elangovan B, Subramanian T, Coleman D, Chinnadurai G (1996) Identification of a cellular protein that specifically interacts with the essential cysteine region of the HIV-1 Tat transactivator. Virology 216:357-366.

Kaneko M, Helfrich-Förster C, Hall JC (1997) Spatial and temporal expression of the period and timeless genes in the developing nervous system of Drosophila: newly identified pacemaker candidates and novel features of clock gene product cycling. J Neurosci 17:6745-6760.

Konopka RJ, Benzer S (1971) Clock mutants of Drosophila melanogaster. Proc Natl Acad Sci U S A 68:2112-2116. 
Kranz D, Dohmesen C, Dobbelstein M (2008) BRCA1 and Tip60 determine the cellular response to ultraviolet irradiation through distinct pathways. J Cell Biol 182:197-213.

Krishnan B, Dryer SE, Hardin PE (1999) Circadian rhythms in olfactory responses of Drosophila melanogaster. Nature 400:375-378.

Kumar S, Mohan A, Sharma VK (2005) Circadian dysfunction reduces lifespan in Drosophila melanogaster. Chronobiol Int 22:641-653.

Kusch T, Florens L, Macdonald WH, Swanson SK, Glaser RL, Yates JR, Abmayr SM, Washburn MP, Workman JL (2004) Acetylation by Tip60 is required for selective histone variant exchange at DNA lesions. Science 306:2084-2087.

Lee C, Bae K, Edery I (1999) PER and TIM inhibit the DNA binding activity of a Drosophila CLOCK-CYC/dBMAL1 heterodimer without disrupting formation of the heterodimer: a basis for circadian transcription. Mol Cell Biol 19:5316-5325.

Looby P, Loudon ASI (2005) Gene duplication and complex circadian clocks in mammals. Trends Genet 21:46-53.

Lorbeck M, Pirooznia K, Sarthi J, Zhu X, Elefant F (2011) Microarray analysis uncovers a role for Tip60 in nervous system function and general metabolism. PLoS ONE 6:e18412.

McDonald MJ, Rosbash M (2001) Microarray analysis and organization of circadian gene expression in Drosophila. Cell 107:567-578.

McGuire SE, Mao Z, Davis RL (2004) Spatiotemporal gene expression targeting with the TARGET and gene-switch systems in Drosophila. Sci STKE 2004:pl6.

Meireles-Filho ACA, Bardet AF, Yáñez-Cuna JO, Stampfel G, Stark A (2014) cis-regulatory requirements for tissue-specific programs of the circadian clock. Curr Biol 24:1-10.

Menet JS, Abruzzi KC, Desrochers J, Rodriguez J, Rosbash M (2010) Dynamic PER repression mechanisms in the Drosophila circadian clock: from on-DNA to off-DNA. Genes Dev 24:358-367.

Mistlberger RE, Antle MC (2011) Entrainment of circadian clocks in mammals by arousal and food. Essays Biochem 49:119-136.

Myers EM, Yu J, Sehgal A (2003) Circadian control of eclosion: interaction between a central and peripheral clock in Drosophila melanogaster. Curr Biol 13:526-533.

Ng FS, Tangredi MM, Jackson FR (2011) Glial cells physiologically modulate clock neurons and circadian behavior in a calcium-dependent manner. Curr Biol 21:625-634. 
Pirooznia SK, Chiu K, Chan MT, Zimmerman JE, Elefant F (2012) Epigenetic regulation of axonal growth of Drosophila pacemaker cells by histone acetyltransferase tip60 controls sleep. Genetics 192:1327-1345.

Preitner N, Damiola F, Luis-Lopez-Molina, Zakany J, Duboule D, Albrecht U, Schibler U (2002) The Orphan Nuclear Receptor REV-ERB $\alpha$ Controls Circadian Transcription within the Positive Limb of the Mammalian Circadian Oscillator. Cell 110:251-260.

Price JL, Blau J, Rothenfluh A, Abodeely M, Kloss B, Young MW (1998) double-time is a novel Drosophila clock gene that regulates PERIOD protein accumulation. Cell 94:83-95.

Renn SC, Park JH, Rosbash M, Hall JC, Taghert PH (1999) A pdf neuropeptide gene mutation and ablation of PDF neurons each cause severe abnormalities of behavioral circadian rhythms in Drosophila. Cell 99:791-802.

Rosato E, Codd V, Mazzotta G, Piccin A, Zordan M, Costa R, Kyriacou CP (2001) Lightdependent interaction between Drosophila CRY and the clock protein PER mediated by the carboxy terminus of CRY. Curr Biol 11:909-917.

Rutila JE, Suri V, Le M, So WV, Rosbash M, Hall JC (1998) CYCLE is a second bHLH-PAS clock protein essential for circadian rhythmicity and transcription of Drosophila period and timeless. Cell 93:805-814.

Sapountzi V, Logan IR, Robson CN (2006) Cellular functions of TIP60. Int J Biochem Cell Biol 38:1496-1509.

Sarthi J, Elefant F (2011) dTip60 HAT activity controls synaptic bouton expansion at the Drosophila neuromuscular junction. PLoS ONE 6:e26202.

Sato TK, Panda S, Miraglia LJ, Reyes TM, Rudic RD, McNamara P, Naik KA, FitzGerald GA, Kay SA, Hogenesch JB (2004) A functional genomics strategy reveals Rora as a component of the mammalian circadian clock. Neuron 43:527-537.

Schirling C, Heseding C, Heise F, Kesper D, Klebes A, Klein-Hitpass L, Vortkamp A, Hoffmann D, Saumweber H, Ehrenhofer-Murray AE (2010) Widespread regulation of gene expression in the Drosophila genome by the histone acetyltransferase dTip60. Chromosoma 119:99-113.

Skopik SD, Pittendrigh CS (1967) Circadian systems, II. The oscillation in the individual Drosophila pupa; its independence of developmental stage. Proc Natl Acad Sci U S A 58:1862-1869.

Suh J, Jackson FR (2007) Drosophila ebony activity is required in glia for the circadian regulation of locomotor activity. Neuron 55:435-447. 
Taylor P, Hardin PE (2008) Rhythmic E-box binding by CLK-CYC controls daily cycles in per and tim transcription and chromatin modifications. Mol Cell Biol 28:4642-4652.

Urban I (2015) The Role of Lysine Acetyltransferase Tip60 in the Murine Hippocampus. GeorgAugust University Goettingen Dissertation Available at: http://ediss.unigoettingen.de/handle/11858/00-1735-0000-0022-6046-5.

Weiss R, Bartok O, Mezan S, Malka Y, Kadener S (2014) Synergistic interactions between the molecular and neuronal circadian networks drive robust behavioral circadian rhythms in Drosophila melanogaster. PLoS Genet 10:e1004252.

Xu S, Wilf R, Menon T, Panikker P, Sarthi J, Elefant F (2014) Epigenetic control of learning and memory in Drosophila by Tip60 HAT action. Genetics 198:1571-1586.

Xu Y, Parmar K, Du F, Price BD, Sun Y (2011) The radioprotective agent WR1065 protects cells from radiation damage by regulating the activity of the Tip60 acetyltransferase. Int $\mathrm{J}$ Biochem Mol Biol 2:295-302.

Yao Z, Shafer OT (2014) The Drosophila circadian clock is a variably coupled network of multiple peptidergic units. Science 343:1516-1520.

Zhao J, Kilman VL, Keegan KP, Peng Y, Emery P, Rosbash M, Allada R (2003) Drosophila clock can generate ectopic circadian clocks. Cell 113:755-766.

Zordan M, Costa R, Macino G, Fukuhara C, Tosini G (2000) CIRCADIAN CLOCKS: WHAT MAKES THEM TICK? Chronobiol Int 17:433-451.

Zordan MA, Sandrelli F (2015) Circadian Clock Dysfunction and Psychiatric Disease: Could Fruit Flies have a Say? Front Neurol 6:80. 


\section{Acknowledgements}

I am very grateful to Prof. Gregor Eichele, for his continuous support and mentorship during my PhD. I am particularly thankful for the liberty I was endowed with to carry out my work and for bringing the best out of me.

I also express my sincere gratitude to members of my thesis committee Prof. André Fiala and PD. Dr. Halyna Shcherbata for their suggestions and critical discussions, during the course of this work. Sincere acknowledgements to the members of the extended examination board Prof. Gregor Bucher, Dr. Manuela Schmidt and Prof. Joerg Grosshans for accepting my invitation to join the committee. I would like to thank Prof. Michael Rosbash, Prof. Amita Sehgal, Prof. Charlotte Helfrich-Foerster, Prof. Ann.E. Ehrenhoeffer-Murray, Dr. Felice Elefant for kindly providing tools and reagents to carry out this work.

I would like to thank my teachers and mentors and I dedicate this thesis for all of them, who shaped my identity and ideas.

I would like to thank the former and present members of the Genes and Behavior department for valuable discussions and productive environment to work. Special thanks to Dr. Lars Geffers for the philosophical discussions, Dr. Inga Urban for her insights on TIP60 and also helping with a German version of thesis summary. Valuable discussions and productive environment provided by Nikolai Petkau, Tahereh Ajam, Dr. Insa Geffers, Dr. Nai-Hua Fruhmans, Dr. Regina Faubel and Dr. Gabriela Whelan and Parth Joshi is greatly acknowledged. Organizational support by Helena Miletic and Christine van den Bogaart are worth a zillion thanks. It would have been technically impossible to work without the humble services of cleanliness and ordering fairy Martina Seebode.

I also thank Dr. Mariya Kucherenko and members of Gene Expression and Signaling group for wonderful help during the early stages of this work and with confocal microscopy. Fly food fairies Karin and Uli made working with flies a pleasant experience. 
I sincerely appreciate the services provided by Otto-Hahn library and Max Planck Digital resources. I am thankful to Peter Boettcher from the workshop for making light tight temperature controlled incubators. I also thank Irene Boettcher-Gajewski and Hartmut Sebesse from media services for their timely help to print my thesis.

It would be incomplete without thanking friends who made my life pleasant than I could think of. Words are not enough to express my thanks to my parents and family members for their unconditional support and constant encouragement.

Last, but not the least, a tribute to all flies that took joy in dedicating their lives for a noble cause.

Vinodh 
Vinodh ILANGOVAN

E-mail: vinodh.ilangovan@mpibpc.mpg.dePhone: (+49) 5512012742 (O)

Current position: Graduate Student, Department of Genes and Behavior, Max Planck Institute for Biophysical Chemistry, Am Fassberg 11, Goettingen, Germany.

\section{Education:}

\begin{tabular}{|l|l|l|}
\hline Degree & University & Year \\
\hline $\begin{array}{l}\text { Ph.D., } \\
\text { Advisor: Prof. Gregor Eichele } \\
\text { Program: Genes and Development }\end{array}$ & $\begin{array}{l}\text { Goettingen Graduate School for Neurosciences, } \\
\text { Biophysics, and Molecular Biosciences, } \\
\text { Georg-August University,Goettingen, } \\
\text { Germany. }\end{array}$ & $\begin{array}{l}\text { Currently } \\
\text { pursuing }\end{array}$ \\
\hline $\begin{array}{l}\text { M.Sc. (5 year Integrated) } \\
\text { Bio-Medical Science }\end{array}$ & $\begin{array}{l}\text { Bharathidasan University, Tiruchirappalli, } \\
\text { India. }\end{array}$ & 2010 \\
\hline
\end{tabular}

\section{Publications}

- IlangovanV, Petkau N, Eichele G. (2013) Understanding the mechanism of biological timekeeping. MPIbpc News 19(9): 1-5.

Peer reviewed

1. Lone SR, Ilangovan V, Murugan M, Sharma VK. (2011) [Short- and long-day responses in the pre-adult developmental duration of two species of Camponotus ants]. Chronobiol Int. 28(2):163-169.

2. Lone SR, Ilangovan V, Murugan M, Sharma VK. (2010) [Circadian resonance in the development of two sympatric species of Camponotus ants].J Insect Physiol. 56(11):1611-1616.

\section{Honors}

- Selected for European PhD School for Chronobiology, Berlin, Germany -17 to 22 September 2012

- Junior Research Fellowship in Life Sciences, CSIR, India -March 2010

- Best research presentation award- DST SERC, Government of India sponsored School in Chronobiology, 25, December 2009 to 7, January 2010

- Rajiv Gandhi Science Talent Research Scholar Award, JNCASR, India- 2006 


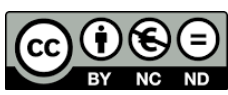

